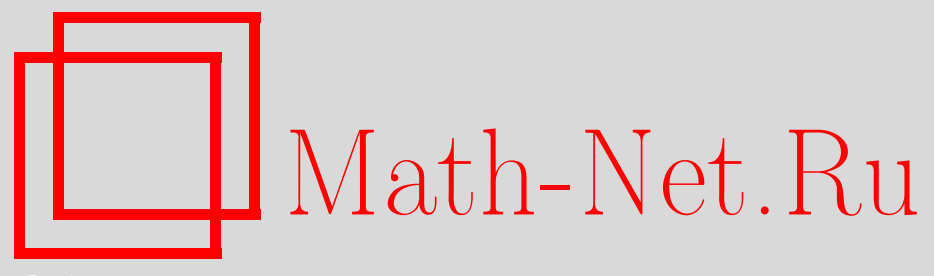

М. Д. Рамазанов, Асимптотически оптимальные решетчатые кубатурные формулы с ограниченным пограничным слоем и свойством ненасыщаемости, Матем. сб., 2013, том 204, номер 7, 71-96

DOI: https://doi.org/10.4213/sm8142

Использование Общероссийского математического портала Math-Net.Ru подразумевает, что вы прочитали и согласны с пользовательским соглашением http://www . mathnet.ru/rus/agreement

Параметры загрузки:

IP : 54.174 .149 .18

26 апреля 2023 г., 18:00:12

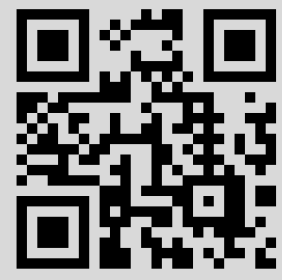




\author{
М. Д. Рамазанов
}

\title{
Асимптотически оптимальные решетчатые кубатурные формулы с ограниченным пограничным слоем и свойством ненасыщаемости
}

В работе описан новый алгоритм построения решетчатых кубатурных формул с ограниченным пограничным слоем

$$
\int_{\Omega} f(x) d x \approx h^{n} \sum_{\substack{k \in \mathbb{Z}^{n} \\ \rho(h k, \Omega) \leqslant C h^{\gamma}}} c_{k}(h) f(h k),
$$

где

$$
\gamma<\frac{1}{2}, \quad c_{k}(h)=1, \quad \text { если } \rho\left(h k, \mathbb{R}^{n} \backslash \Omega\right) \geqslant C h^{\gamma} .
$$

Эти формулы не насыщаемы (по Бабанко) не только по порядку, но и по свойству асимптотической оптимальности на $W_{2}^{m}$-пространствах, $m \in(n / 2, \infty)$. Большая часть результатов сохраняется и на $W_{2}^{\mu}\left(\mathbb{R}^{n}\right)$ пространствах с гипоэллиптическим символом гладкости $\mu$.

Библиография: 6 названий.

Ключевые слова: кубатурные формулы, оптимизация, ненасыщаемый алгоритм.

DOI: $10.4213 / \mathrm{sm} 8142$

\section{§ 1. Введение}

Решетчатые кубатурные формулы дают приближенные значения интегралов $\int_{\Omega} f(x) d x$ по многомерным областям $\Omega \subset \mathbb{R}^{n}$ в виде линейных комбинаций значений подынтегральной функции в узлах выбранной решетки

$$
\mathscr{K}_{N} f \equiv\left|\operatorname{det} H_{N}\right| \sum_{\substack{k \in \mathbb{Z}^{n} \\ H_{N} k \in \Omega}} c_{k}(N) f\left(H_{N} k\right),
$$

где

$$
H_{N}-(n \times n) \text {-матрица, } \quad \operatorname{det} H_{N}=\frac{|\Omega|}{N} \text {. }
$$

В конце 60-х годов прошлого века С. Л. Соболев (см. [1], [2]) предложил алгоритм получения формул высокой точности, объединяющий подходы функционального анализа и алгебраический. 
А именно, гладкость интегрантов задавалась принадлежностью их конкретному банаховому функциональному пространству $B(\Omega) \subset C$, качество формулы определялось нормой функционала погрешности

$$
l_{N}^{\Omega}: f \rightarrow \int_{\Omega} f(x) d x-\mathscr{K}_{N} f
$$

с оптимизацией по коэффициентам $\left\{c_{k}\right\}$ :

$$
l_{N}^{\Omega, \mathrm{opt}}=\arg \min _{l_{N}^{\Omega},\left\{c_{k}\right\}}\left\|l_{N}^{\Omega} \mid[B(\Omega)]^{*}\right\| .
$$

А сам алгоритм основывался на замене интеграла по области интегрирования суммой интегралов по всем элементарным ячейкам решетки

$$
Q_{N, k}=\left\{x \mid H_{N}^{-1} x-k \in[0,1)^{n}\right\},
$$

вблизи границы области $Q_{N, k} \cup \Omega$ Интегралы по элементарным ячейкам приближались с помощью алгебраических формул с теми же узлами, точно интегрирующих многочлены до некоторой степени $M$, связанной с гладкостью интегрантов. С.Л. Соболев установил, что при $N \rightarrow \infty$ последовательность функционалов погрешностей таких формул обладает свойством асимптотической оптимальности

$$
\left\|l_{N}^{\Omega, \text { as }}\left|[B(\Omega)]^{*}\|=\| l_{N}^{\Omega, \text { opt }}\right|[B(\Omega)]^{*}\right\| \cdot(1+o(1))
$$

на пространствах интегрантов $B(\Omega)=L_{2}^{(m)}(\Omega)$ с полунормой

$$
\left\|f \mid L_{2}^{(m)}(\Omega)\right\|=\left[\int_{\Omega}\left(\sum_{|\alpha|=m} \frac{|\alpha| !}{\alpha !}\left|D^{\alpha} f(x)\right|^{2}\right) d x\right]^{1 / 2} .
$$

Свойство асимптотической оптимальности было установлено и для многих других банаховых пространств, обычно употребляемых в вычислительной математике (см. [2]-[4]). Важнейшей особенностью соболевского алгоритма является свойство пограничного слоя: узлам, удаленным от границы области на расстояние больше $O\left(N^{-1 / n}\right)$, соответствуют одинаковые коэффициенты:

$$
\exists L, \forall k, N \quad \operatorname{dist}\left(H_{N} k, \partial \Omega\right)>L N^{-1 / n} \quad \Longrightarrow \quad c_{k} \equiv 1 .
$$

Это на порядок уменьшает объем вычислительной работы и позволяет установить для таких формул фактическую эквивалентность асимптотической и порядковой оптимальности на соболевских пространствах $W_{p}^{m}$ (см. [3]).

Решетчатые кубатурные формулы, коэффициенты которых ограничены равномерно по $k$ и $N$ и удовлетворяют условию (1.3), были названы формулами с ограниченным пограничным слоем (ОПС-формулы; см. [4]).

Заложенная в алгоритме алгебраическая точность локальных формул на всех многочленах до некоторой степени $M$ проявилась в условной "асимптотической ненасыщаемости" алгоритма. А именно, построенная последовательность кубатурных формул остается асимптотически (при $N \rightarrow \infty$ ) оптимальной 
на каждом пространстве $W_{p}^{m}$ с $m \in(n / p, M)$, т.е. в ослабленной форме удовлетворяет введенному К. И. Бабенко определению ненасыщаемости. (У К. И. Бабенко в [5], [6] универсальна порядковая оптимальность без ограничения сверху на гладкость интегрантов.) Ненасыщаемость - важное свойство вычислительных алгоритмов, позволяющее созданным по ним программам автоматически настраиваться на проявляющиеся характеристики гладкостей параметров задач, обеспечивая наилучшие скорости сходимостей аппроксимаций.

В настоящей работе мы ограничиваемся кубической решеткой узлов $H=h I_{n}$ с единичной матрицей $I_{n}, N=|\Omega| h^{-n}$, и предлагаем новый алгоритм решетчатых кубатурных формул

$$
\mathscr{K}_{h} f=h^{n} \sum_{k \in \mathbb{Z}^{n}} c_{k} f(h k)
$$

обладающих свойством ограниченного пограничного слоя и являющихся асимптотически ненасыщаемыми на всех пространствах $W_{2}^{m}\left(\mathbb{R}^{n}\right)$ с $m>n / 2$.

Сначала выводим формулу коэффициентов оптимальных кубатурных формул для интегралов более общего вида

$$
\mathscr{I}^{\varphi} f=\int_{\mathbb{R}^{n}} \varphi(x) f(x) d x
$$

и на более общих пространствах $W_{2}^{\mu}\left(\mathbb{R}^{n}\right)$. Норма их задается равенством

$$
\left\|f \mid W_{2}^{\mu}\left(\mathbb{R}^{n}\right)\right\|=\sqrt{\int_{\mathbb{R}^{n}}|\tilde{f}(\xi) \mu(2 \pi i \xi)|^{2} d \xi}
$$

с произвольной функцией $\mu$. Затем, ограничиваясь символами $\mu$, обладающими свойством гипоэллиптичности, упрощаем выражения оптимальных коэффициентов, отсекая слагаемые, порядки которых пренебрежимо малы по сравнению с главным членом.

Таким образом, мы приходим к формуле коэффициентов асимптотически оптимальной кубатурной формулы с ограниченным пограничным слоем.

Далее мы ограничиваемся частным случаем с весовой функцией $\varphi$, являющейся характеристической функцией области интегрирования, и изотропной функцией $\mu, \mu(2 \pi i \xi)=\left(1+|2 \pi \xi|^{2}\right)^{m / 2}$. Упрощая формулу коэффициентов в пограничном слое, получаем коэффициенты асимптотически ненасыщаемой формулы.

\section{§ 2. Формула коэффициентов решетчатой кубатурной формулы, оптимально аппроксимирующей интегралы с весовыми функциями}

Определим пространство $W_{2}^{\mu}\left(\mathbb{R}^{n}\right)$ как пополнение в норме (1.4) множества $C_{0}^{\infty}\left(\mathbb{R}^{n}\right)$ финитных бесконечно дифференцируемых функций. Пусть

$$
\widetilde{f}(\xi) \equiv \int_{\mathbb{R}^{n}} f(x) e^{-2 \pi i x \xi} d x
$$


есть преобразование Фурье функции $f ; \mu: \mathbb{R}^{n} \rightarrow \mathbb{C}-$ функция с конечным интегралом

$$
\int_{\mathbb{R}^{n}} \frac{d \xi}{|\mu(2 \pi i \xi)|^{2}}<\infty
$$

что обеспечивает вложение $W_{2}^{\mu}\left(\mathbb{R}^{n}\right)$ в пространство непрерывных функций $C\left(\mathbb{R}^{n}\right)$.

Будем полагать $\mu$ бесконечно дифференцируемой, четной, не обращающейся в нуль нигде и равной единице в начале координат, $\mu(0)=1$. Кроме того, мы полагаем, что функция $\mu$ имеет не более чем степенной порядок роста. Точнее:

$$
\exists m^{\prime \prime} \geqslant m^{\prime}>0, \quad \exists C>0: \quad \forall \xi \quad \frac{1+|\xi|^{m^{\prime}}}{C} \leqslant|\mu(2 \pi i \xi)| \leqslant\left(1+|\xi|^{m^{\prime \prime}}\right) \cdot C .
$$

Пусть еще

$$
\int \frac{d \xi}{1+|\xi|^{2 m^{\prime}}}<\infty
$$

Мы считаем $\mu(2 \pi i \xi)$ символом гипоэллиптического псевдодифференциального оператора

$$
\mu(D): f(x) \rightarrow \mu(D) f(x)=\int \tilde{f}(\xi) \mu(2 \pi i \xi) e^{2 \pi i \xi x} d \xi .
$$

Гипоэллиптичность означает выполнение условия

$$
\exists \rho \in(0,1] \quad \forall \alpha \in \mathbb{Z}_{+}^{n} \quad \exists C_{\alpha}: \quad \forall \xi \in \mathbb{R}^{n} \quad\left|\frac{D^{\alpha} \mu(2 \pi i \xi)}{\mu(2 \pi i \xi)}\right| \leqslant \frac{C_{\alpha}}{(1+|\xi|)^{\rho|\alpha|}} .
$$

В этой работе основным частным случаем будет $\mu(2 \pi i \xi)=\left(1+|2 \pi \xi|^{2}\right)^{m / 2}$, $m>n / 2$. Обычное обозначение этого пространства, взятого в одной из эквивалентных нормировок, $W_{2}^{m}\left(\mathbb{R}^{n}\right)$.

Как уже было указано, качество кубатурной формулы характеризуется $\left[W_{2}^{\mu}\left(\mathbb{R}^{n}\right)\right]^{*}$-нормой ее функционала погрешности $l_{h}^{\varphi}: f \rightarrow \mathscr{I}^{\varphi} f-\mathscr{K}_{h} f$. Здесь $\mathscr{I}^{\varphi}: f \rightarrow \int_{\mathbb{R}^{n}} \varphi(x) f(x) d x$ и весовая функция предполагается гладкой в $\bar{\Omega} \equiv$ $\operatorname{supp} \varphi, \varphi \in C^{\infty}(\bar{\Omega})$. Оптимальные коэффициенты определяются условием

$$
\left\{c_{k}^{\mathrm{opt}}(h)\right\}=\arg \min _{\left\{c_{k}\right\}}\left\|\mathscr{I}^{\varphi}-\mathscr{K}_{h} \mid\left[W_{2}^{\mu}\left(\mathbb{R}^{n}\right)\right]^{*}\right\| .
$$

Следует заметить, что мы берем кубатурную формулу с суммированием по всем узлам $\left\{h k \mid k \in \mathbb{Z}^{n}\right\}$ и требуем, чтобы функционал $\mathscr{K}_{h}$ принадлежал пространству $\left[W_{2}^{\mu}\left(\mathbb{R}^{n}\right)\right]^{*}$. Функционал погрешности оптимальной кубатурной формулы $l_{h}^{\varphi, \text { opt }}=\mathscr{I}^{\varphi}-\mathscr{K}_{h}^{\text {opt }}$ должен удовлетворять условию

$$
\left\|l_{h}^{\varphi, \text { opt }}\left|\left[W_{2}^{\mu}\left(\mathbb{R}^{n}\right)\right]^{*}\left\|=\inf _{\left\{c_{k}\right\}}\right\| \mathscr{I}^{\varphi}-\mathscr{K}_{h}\right|\left[W_{2}^{\mu}\left(\mathbb{R}^{n}\right)\right]^{*}\right\| .
$$

В гильбертовом пространстве шар слабо компактен и слабо замкнут. Поэтому мы можем описанную выше операцию инфинума нормы в $\left[W_{2}^{\mu}\left(\mathbb{R}^{n}\right)\right]^{*}$ заменить 
на нахождение минимума нормы. При этом аргумент минимума - единственный и оптимальный функционал погрешности - однозначно определен.

Первой нашей целью является получение формул оптимальных коэффициентов и выписывание их асимптотики по параметру $h$ до такого порядка, который позволит заменить оптимальную кубатурную формулу асимптотически оптимальной $\mathscr{K}_{h}^{\text {as }} f$ (см. (1.2)) с более простым алгоритмом вычисления коэффициентов.

Коэффициенты кубатурных формул естественно считать комплексными, $c_{k}=c_{k}^{1}+i c_{k}^{2}$. Поскольку квадрат нормы функционала погрешности является положительным многочленом второй степени от $\left\{c_{k}^{1}, c_{k}^{2}\right\}_{k \in \mathbb{Z}^{n}}$, уравнения, которым подчиняются оптимальные коэффициенты, задаются формулами

$$
0=\frac{\partial}{\partial c_{k}^{1}}\left\|l_{h}^{\varphi, \text { opt }}\left|\left[W_{2}^{\mu}\left(\mathbb{R}^{n}\right)\right]^{*}\left\|^{2}=\frac{\partial}{\partial c_{k}^{2}}\right\| l_{h}^{\varphi, \text { opt }}\right|\left[W_{2}^{\mu}\left(\mathbb{R}^{n}\right)\right]^{*}\right\|^{2} .
$$

Прямыми вычислениями они сводятся к одному равенству

$$
0=\int_{\mathbb{R}^{n}} \frac{e^{2 \pi i \xi h j}}{|\mu(2 \pi i \xi)|^{2}}\left[\widetilde{\varphi}(\xi)-h^{n} \sum_{k \in \mathbb{Z}^{n}} c_{k}^{\mathrm{opt}} e^{-2 \pi i \xi h k}\right] d \xi \quad \forall j \in \mathbb{Z}^{n},
$$

где

$$
\widetilde{\varphi}(\xi)=\int_{\mathbb{R}^{n}} \varphi(x) e^{-2 \pi i x \xi} d x .
$$

Сходимость этого интеграла обеспечивается нашими основными предположениями о весовой функции $\varphi$. А именно, считаем носитель функции замыканием ограниченной $n$-мерной области $\Omega$ с гладкой границей $\Gamma$, а саму функцию $\varphi$ бесконечно дифференцируемой в $\bar{\Omega}$,

$$
\operatorname{supp} \varphi \equiv \bar{\Omega} \Subset \mathbb{R}^{n}, \quad \partial \Omega \equiv \Gamma \in C^{\infty}, \quad \varphi \in C^{\infty}(\bar{\Omega}) .
$$

Займемся решением уравнений оптимальных коэффициентов.

Интеграл по $\xi$ разобьем на отдельные блоки:

$$
\xi=\frac{t}{h}+\eta, \quad t \in \mathbb{Z}^{n}, \quad \eta \in \frac{Q}{h}, \quad Q=\left[-\frac{1}{2}, \frac{1}{2}\right)^{n}, \quad \int_{\mathbb{R}^{n}} d \xi=\sum_{t \in \mathbb{Z}^{n}} \int_{Q / h} d \eta .
$$

Теперь

$$
\begin{gathered}
h^{n} \sum_{k \in \mathbb{Z}^{n}} c_{k}^{\mathrm{opt}} \int_{Q / h} e^{2 \pi i \eta(j-k) h} \sum_{t \in \mathbb{Z}^{n}} \frac{1}{|\mu(2 \pi i(t / h+\eta))|^{2}} d \eta \\
=\int_{Q / h} e^{2 \pi i \eta j h} \sum_{t \in \mathbb{Z}^{n}} \frac{\widetilde{\varphi}(t / h+\eta)}{|\mu(2 \pi i(t / h+\eta))|^{2}} d \eta .
\end{gathered}
$$

Сделаем замену переменных $h \eta=\zeta$ :

$$
\begin{aligned}
\sum_{k \in \mathbb{Z}^{n}} & c_{k}^{\mathrm{opt}} \int_{Q} e^{2 \pi i \zeta(j-k)} \sum_{t \in \mathbb{Z}^{n}} \frac{1}{|\mu(2 \pi i(t+\zeta) / h)|^{2}} d \zeta \\
= & h^{-n} \int_{Q} e^{2 \pi i \zeta j} \sum_{t \in \mathbb{Z}^{n}} \frac{\widetilde{\varphi}((t+\zeta) / h)}{|\mu(2 \pi i(t+\zeta) / h)|^{2}} d \zeta .
\end{aligned}
$$


Обе части равенства умножим на $e^{-2 \pi i \sigma j}$ и просуммируем по $j \in \mathbb{Z}^{n}$ :

$$
\begin{gathered}
\sum_{j \in \mathbb{Z}^{n}} e^{-2 \pi i \sigma j} \int_{Q}\left[\sum_{k \in \mathbb{Z}^{n}} c_{k}^{\mathrm{opt}} e^{-2 \pi i \zeta k} \sum_{t \in \mathbb{Z}^{n}} \frac{1}{|\mu(2 \pi i(t+\zeta) / h)|^{2}}\right] e^{2 \pi i \zeta j} d \zeta \\
=\sum_{j \in \mathbb{Z}^{n}} e^{-2 \pi i \sigma j} \int_{Q} e^{2 \pi i \zeta j}\left[h^{-n} \sum_{t \in \mathbb{Z}^{n}} \frac{\widetilde{\varphi}((t+\zeta) / h)}{|\mu(2 \pi i(t+\zeta) / h)|^{2}}\right] d \zeta .
\end{gathered}
$$

Заменим $j$ на $-j$ и заметим, что в правой и левой частях равенства стоят ряды Фурье по $j$ с коэффициентами от функций, заключенных в квадратные скобки. Значит, равны сами функции. Отсюда следует

$$
\sum_{k \in \mathbb{Z}^{n}} c_{k}^{\mathrm{opt}} e^{-2 \pi i \zeta k}=h^{-n} \frac{\sum_{t \in \mathbb{Z}^{n}} \widetilde{\varphi}((t+\zeta) / h) /|\mu(2 \pi i(t+\zeta) / h)|^{2}}{\sum_{s \in \mathbb{Z}^{n}}|\mu(2 \pi i(s+\zeta) / h)|^{-2}} .
$$

Заменим $\zeta$ на $-\zeta$ и заметим, что $c_{k}^{\text {opt }}$ - это коэффициенты ряда Фурье функции, стоящей в правой части равенства.

По формулам для коэффициентов Фурье получаем

$$
c_{k}^{\mathrm{opt}} \equiv c_{k}^{\mathrm{opt}}(h)=\int_{Q} e^{-2 \pi i \zeta k} h^{-n} \frac{\sum_{t \in \mathbb{Z}^{n}} \widetilde{\varphi}((t-\zeta) / h) /|\mu(2 \pi i(t-\zeta) / h)|^{2}}{\sum_{s \in \mathbb{Z}^{n}}|\mu(2 \pi i(s-\zeta) / h)|^{-2}} d \zeta .
$$

Если ввести функцию непрерывного аргумента (и заменить $\zeta$ на $-\zeta$ )

$$
C^{\mathrm{opt}}(x, h)=\int_{Q} e^{2 \pi i \zeta x / h} h^{-n} \frac{\sum_{t \in \mathbb{Z}^{n}} \widetilde{\varphi}((t+\zeta) / h) /|\mu(2 \pi i(t+\zeta) / h)|^{2}}{\sum_{s \in \mathbb{Z}^{n}}|\mu(2 \pi i(s+\zeta) / h)|^{-2}} d \zeta,
$$

TO

$$
c_{k}^{\mathrm{opt}}(h)=\left.C^{\mathrm{opt}}(x, h)\right|_{x=h k} \quad \forall k \in \mathbb{Z}^{n} .
$$

Назовем $C^{\text {opt }}(x, h)$ функцией оптимальных коэффициентов.

Сформулируем полученный результат.

Теорема 1. Равенства $(2.3),(2.4)$ задают коэфбиииенты кубатурной формуль, оптимальной на пространстве $W_{2}^{\mu}\left(\mathbb{R}^{n}\right)$.

ЗАмечАниЕ. Решетчато наилучшей формулой называется результат оптимизации не только по коэффициентам, но и по всевозможным решеткам узлов.

$$
\widehat{H}_{N} \equiv H_{N}^{\text {best }}=\arg \min _{\left|\operatorname{det} H_{N}\right|=|\Omega| / N}\left\|l_{N}^{\Omega, \text { opt }} \mid\left[W_{2}^{\mu}\left(\mathbb{R}^{n}\right)\right]^{*}\right\| \quad \forall N .
$$

Произвольное решетчатое расположение узлов можно свести к кубическому заменой переменных $x=H_{N}^{-1}\left|\operatorname{det} H_{N}\right|^{1 / n} \cdot y$. Поэтому

$$
C^{\text {best }}(x, N) \equiv \int_{Q} e^{2 \pi i \zeta x / h} h^{-n} \frac{\sum_{t \in \mathbb{Z}^{n}} \widetilde{\varphi}_{N}((t-\zeta) / h) /\left|\mu\left(2 \pi i \widehat{H}_{N}^{*-1}(t+\zeta)\right)\right|^{2}}{\sum_{s \in \mathbb{Z}^{n}}\left|\mu\left(2 \pi i \widehat{H}_{N}^{*-1}(s+\zeta)\right)\right|^{-2}} d \zeta,
$$

где $h \equiv\left|\operatorname{det} \widehat{H}_{N}\right|^{1 / n}, \varphi_{N}(x) \equiv \varphi\left(\widehat{H}_{N}^{-1} h x\right), \widetilde{\varphi}_{N}(\xi) \equiv \widetilde{\varphi}\left(\widehat{H}^{*} h \xi\right)$. 


\section{§ 3. Оценка порядка точности вычислений при получении функции асимптотически оптимальных коэффициентов}

Асимптотическая оптимальность функционала погрешности означает, что отличие его нормы от нормы оптимального функционала есть величина бесконечно малая по сравнению с нормой асимптотически оптимального функционала. В наших гильбертовых пространствах оценка разности норм может быть заменена на оценку нормы разности оптимального и асимптотически оптимального функционалов. Покажем это, обозначая для краткости $\left[W_{2}^{\mu}\left(\mathbb{R}^{n}\right)\right]^{*}$-норму $l$ через $\|l\|_{*}$ и эквивалентность порядков знаком こ:

$$
\begin{aligned}
0 & \leqslant \frac{\left\|l_{h}^{\varphi, \text { as }}\right\|_{*}-\left\|l_{h}^{\varphi, \mathrm{opt}}\right\|_{*}}{\left\|l_{h}^{\varphi, \mathrm{as}}\right\|_{*}}=\frac{\left\|l_{h}^{\varphi, \mathrm{as}}\right\|_{*}^{2}-\left\|l_{h}^{\varphi, \mathrm{opt}}\right\|_{*}^{2}}{\left(\left\|l_{h}^{\varphi, \mathrm{as}}\right\|_{*}+\left\|l_{h}^{\varphi, \mathrm{opt}}\right\|_{*}\right)\left\|l_{h}^{\varphi, \mathrm{as}}\right\|_{*}} \\
& \asymp\left(\frac{\left\|l_{h}^{\varphi, \mathrm{as}}-l_{h}^{\varphi, \mathrm{opt}}\right\|_{*}}{\left\|l_{h}^{\varphi, \mathrm{as}}\right\|_{*}}\right)^{2},
\end{aligned}
$$

и это должно быть $о(1)$ при $h \rightarrow 0$.

ЛЕмма 1. Если $\varphi \not \equiv 0$, mo

$$
\begin{aligned}
\left\|l_{h}^{\varphi, \text { as }} \mid\left[W_{2}^{\mu}\left(\mathbb{R}^{n}\right)\right]^{*}\right\| & \geqslant\left\|l_{h}^{\varphi, \text { opt }} \mid\left[W_{2}^{\mu}\left(\mathbb{R}^{n}\right)\right]^{*}\right\| \\
& \geqslant \text { const } \cdot \sqrt{\sum_{k \in \mathbb{Z}^{n} \backslash 0} \frac{1}{|\mu(2 \pi i k / h)|^{2}}} .
\end{aligned}
$$

ДокАЗАТЕЛЬСтво. Очевидно, для любого функционала погрешности и любой функции $u \in W_{2}^{\mu}\left(\mathbb{R}^{n}\right)$

$$
\left\|l_{h}^{\varphi} \mid\left[W_{2}^{\mu}\left(\mathbb{R}^{n}\right)\right]^{*}\right\| \geqslant \frac{\left|\left(l_{h}^{\varphi}, u\right)\right|}{\left\|u \mid W_{2}^{\mu}\left(\mathbb{R}^{n}\right)\right\|} .
$$

Подберем подходящую функцию $u$. Наша функция $u$ будет неотрицательной и обращающейся в нуль в каждом узле $h k$. Поэтому

$$
\left|\left(l_{h}^{\varphi}, u\right)\right|=\left|\int_{\Omega} \varphi(x) u(x) d x\right| .
$$

Так как $\varphi$ непрерывна и не является тождественным нулем, то она отделена от нуля на некотором множестве $\omega,\left.|\varphi(x)|\right|_{x \in \omega} \geqslant c>0$.

Можем полагать, что $\omega$ имеет форму прямоугольного параллелепипеда $\omega=$ $\prod_{j=1}^{n}\left[a_{j}, b_{j}\right]$, целиком помещающегося в единичном кубе $[-1 / 2,1 / 2)^{n}$.

Выделим эту область срезающей функцией. Пусть $\varkappa(t)$ - "гладкая ступенька", т.е. бесконечно дифференцируемая, неотрицательная, монотонная, с графиком, симметричным относительно точки $(1 / 2,1 / 2)$, функция $\varkappa \in C^{\infty}$, $\varkappa^{\prime}(t) \geqslant 0, \varkappa(t)=0$ при $t \leqslant 0$ и $\varkappa(t)=1$ при $t \geqslant 1, \varkappa(1 / 2-t)=\varkappa(1 / 2+t)$. Положим

$$
\theta(x)=\prod_{j=1}^{n} \varkappa\left(\frac{x_{j}-a_{j}}{\tau}\right) \varkappa\left(\frac{b_{j}-x_{j}}{\tau}\right),
$$


подобрав параметр $\tau$ так, чтобы множество, на котором $\theta(x)=1$, было достаточно массивным:

$$
\int \theta(x) d x=\prod_{j=1}^{n}\left(b_{j}-a_{j}-\tau\right) \geqslant \frac{1}{2} \prod_{j=1}^{n}\left(b_{j}-a_{j}\right)=\frac{|\operatorname{supp} \theta|}{2} .
$$

Возьмем $u(x)=\theta(x)\left[v_{h}(x)-v_{h}(0)\right]$, где $v_{h}(x)$ - экстремальная функция однородного функционала погрешности

$$
\begin{aligned}
l_{h}^{1}(x) & =\chi_{Q_{h}}(x)-h^{n} \sum_{h k \in Q_{h}} \delta(x-h k), \\
Q_{h} & =[-H h, H h)^{n} \text { с } H=\left[\frac{[1 / h]+1}{2}\right]
\end{aligned}
$$

(квадратные скобки последней формулы означают целую часть числа).

$$
\left(l_{h}^{1}, v_{h}\right)=\left\|l_{h}^{1}\left|\left(\widetilde{W}_{2}^{\mu}\left(Q_{h}\right)\right)^{*}\|, \quad\| v_{h}\right| \widetilde{W}_{2}^{\mu}\left(Q_{h}\right)\right\|=1 .
$$

Пространство $\widetilde{W}_{2}^{\mu}\left(Q_{h}\right)$ состоит из периодических с основным периодом $Q_{h}$ функций $f(x)=\sum_{k \in \mathbb{Z}^{n}} f_{k} e^{2 \pi i k x /(2 H h)}$ с $f_{k}=(2 H h)^{-n} \int_{Q_{h}} f(y) e^{-2 \pi i y /(2 H h)} d y$ и конечной нормой

$$
\left\|f \mid \widetilde{W}_{2}^{\mu}\left(Q_{h}\right)\right\|=(2 H h)^{-n / 2}\left(\sum_{k \in \mathbb{Z}^{n}}\left|f_{k} \mu\left(\frac{2 \pi i k}{2 H h}\right)\right|^{2}\right)^{1 / 2} .
$$

Как известно (см. [4]), экстремальная функция $v_{h}(x)$ имеет еще и маленький период $h\left(v_{h}(x+h k)=v_{h}(x)\right.$ для всех $\left.k \in \mathbb{Z}^{n}\right)$, вещественна и всюду не меньше своего значения в нуле, $v_{h}(x)-v_{h}(0) \geqslant 0$.

Таким образом,

$$
\left\|l_{h}^{\varphi} \mid\left(W_{2}^{\mu}\left(\mathbb{R}^{n}\right)\right)^{*}\right\| \geqslant c \sum_{\substack{j \in \mathbb{Z}^{n} \\ \theta(h j)=1}} \int_{h Q} \frac{\left[v_{h}(x-h j)-v_{h}(0)\right]}{\left\|\left(v_{h}-v_{h}(0)\right) \theta \mid W_{2}^{\mu}\left(\mathbb{R}^{n}\right)\right\|} d x .
$$

Числитель этой дроби есть

$$
\begin{aligned}
& \sum_{\substack{j \in \mathbb{Z}^{n} \\
\theta(h j)=1}} \int_{h Q}\left[v_{h}(x-h j)-v_{h}(0)\right] d x \\
& =\frac{|\{x \mid \theta(x)=1\}|}{h^{n}} \int_{h Q}\left[v_{h}(x)-v_{h}(0)\right] d x \\
& =|\{x \mid \theta(x)=1\}|(2 H h)^{-n}\left(l_{h}^{1}, v_{h}-v_{h}(0)\right) \\
& =|\{x \mid \theta(x)=1\}|(2 H h)^{-n}\left(l_{h}^{1}, v_{h}\right) \\
& =|\{x \mid \theta(x)=1\}|(2 H h)^{-n} \| l_{h}^{1} \mid\left(\widetilde{\left.W_{2}^{\mu}\left(Q_{h}\right)\right)^{*} \|}\right. \\
& =|\{x \mid \theta(x)=1\}|(1+O(h)) \cdot \sqrt{\sum_{s \in \mathbb{Z}^{n} \backslash 0} \frac{1}{|\mu(2 \pi i s / h)|^{2}}} .
\end{aligned}
$$


При оценке знаменателя учтем известную эквивалентность норм $W_{2}^{\mu}\left(\mathbb{R}^{n}\right)$ и $\widetilde{W}_{2}^{\mu}\left(Q_{h}\right)$ для функций, сосредоточенных в $\operatorname{int} Q_{h}$, и гипоэллиптических символов $\mu$. Благодаря этому получим

$$
\begin{aligned}
\left\|\left(v_{h}-v_{h}(0)\right) \theta \mid W_{2}^{\mu}\left(\mathbb{R}^{n}\right)\right\| & \leqslant \text { const } \cdot\left\|\left(v_{h}-v_{h}(0)\right) \theta \mid \widetilde{W}_{2}^{\mu}\left(Q_{h}\right)\right\| \\
& \leqslant \text { const } \cdot\left\|v_{h}-v_{h}(0) \mid \widetilde{W}_{2}^{\mu}\left(Q_{h}\right)\right\| \leqslant \text { const. }
\end{aligned}
$$

Итак,

$$
\left\|l_{h}^{\varphi} \mid\left(W_{2}^{\mu}\left(\mathbb{R}^{n}\right)\right)^{*}\right\| \geqslant \mathrm{const} \cdot \sqrt{\sum_{s \in \mathbb{Z}^{n}} \frac{1}{|\mu(2 \pi i s / h)|^{2}} .}
$$

СледСтвиЕ. Для пространств $W_{2}^{m}$ формула (3.1) означает, что

$$
\left\|l_{h}^{\varphi} \mid\left(W_{2}^{m}\left(\mathbb{R}^{n}\right)\right)^{*}\right\| \geqslant \text { const } \cdot h^{m} .
$$

Можно показать, что для оптимального функционала выполняется и обратная оценка

$$
\left\|l_{h}^{\varphi, \text { opt }} \mid\left(W_{2}^{m}\left(\mathbb{R}^{n}\right)\right)^{*}\right\| \leqslant \text { const } \cdot|\operatorname{supp} \varphi|^{1 / 2} h^{m} .
$$

\section{§ 4. Упрощение формулы коэффициентов для узлов, достаточно удаленных от области интегрирования}

Возвращаясь от переменных $t \in \mathbb{Z}^{n}, \zeta \in Q$ к $\xi=(t+\zeta) / h$, можем записать

$$
C^{\mathrm{opt}}(x, h)=\int_{\Omega} \varphi(y) \int_{\mathbb{R}^{n}} \frac{e^{2 \pi i(x-y) \xi}}{|\mu(2 \pi i \xi)|^{2}} \nu(\xi, h) d \xi d y,
$$

где

$$
\nu(\xi, h)=\frac{1}{\sum_{s \in \mathbb{Z}^{n}}|\mu(2 \pi i(s / h+\xi))|^{-2}} .
$$

Функция $\xi \rightarrow \nu(\xi, h)$ бесконечно дифференцируемая, периодическая с основным периодом $Q / h$.

Мы накладываем условие

$$
\operatorname{dist}(x, \Omega) \geqslant \psi(h)=o(1) \quad \text { при } h \rightarrow 0 .
$$

Позже уточним порядок малости $\psi(h)$ (см. формулу (4.5)) и воспользуемся этим, чтобы упростить выражение $C^{\mathrm{opt}}(x, h)$.

Зададим финитную функцию

$$
\sigma(\xi, \delta)=\prod_{j=1}^{n}\left[1-\varkappa\left(\left|\xi_{j}\right| h^{\delta}-2\right)\right]
$$

с некоторым $\delta>0$. Рассмотрим часть функции $C^{\text {opt }}(x, h)$ с интегралом по $\xi$, взятым вне $O\left(h^{-\delta}\right)$-окрестности начала координат переменных $\xi$ :

$$
\begin{gathered}
C_{\delta}^{\mathrm{opt}}(x, h) \equiv \int_{\Omega} \varphi(y) \int_{\mathbb{R}^{n}} \frac{e^{2 \pi i(x-y) \xi}}{|\mu(2 \pi i \xi)|^{2}} \nu(\xi, h)[1-\sigma(\xi, \delta)] d \xi d y, \\
\operatorname{supp} D_{\xi}(1-\sigma) \subseteq\left\{\xi\left|\max _{j}\right| \xi_{j} \mid \leqslant \frac{3}{h^{\delta}}\right\} \backslash\left\{\xi\left|\max _{j}\right| \xi_{j} \mid \leqslant \frac{2}{h^{\delta}}\right\} .
\end{gathered}
$$


Теперь подготовим интегрирование по частям в интеграле по $\xi$ с вычислением первообразных от $e^{2 \pi i(x-y) \xi} \nu(\xi, h)$. Пусть будет

$$
\nu(\xi, h) e^{2 \pi i(x-y) \xi} \equiv \Delta_{\xi}^{N}\left(e^{2 \pi i(x-y) \xi} f(\xi)\right),
$$

где $\Delta_{\xi}$ - оператор Лапласа.

Вычислим $f(\xi)$ как решение этого уравнения. Пусть

$$
\begin{aligned}
I & \equiv \Delta_{\xi}^{N}(g(\xi) f(\xi))=\Delta_{\xi}^{N}\left[F_{x \rightarrow \xi}^{-1}\left(\int_{\mathbb{R}^{n}} \widetilde{g}(t) \tilde{f}(x-t) d t\right)\right](\xi) \\
& =\Delta_{\xi}^{N} \int e^{2 \pi i x \xi} \int \widetilde{g}(t) \widetilde{f}(x-t) d t d x \\
& =\iint(-2 \pi i|x|)^{2 N} \widetilde{g}(t) \widetilde{f}(x-t) e^{2 \pi i x \xi} d t d x .
\end{aligned}
$$

Для упрощения формулы возьмем $N$ четным числом:

$$
\begin{gathered}
I=(2 \pi)^{2 N} \iint\left(|t|^{2}+2(t, x-t)+|x-t|^{2}\right)^{N} \widetilde{g}(t) \widetilde{f}(x-t) e^{2 \pi i x \xi} d t d x \\
=(2 \pi)^{2 N} \iint e^{2 \pi i x \xi} \sum_{\alpha_{1}+\alpha_{2}+\alpha_{3}=N} C_{N}^{\alpha}|t|^{2 \alpha_{1}}(2(t, x-t))^{\alpha_{2}}|x-t|^{2 \alpha_{3}} \widetilde{g}(t) \widetilde{f}(x-t) d t d x \\
(2(t, x-t))^{\alpha_{2}}=2^{\alpha_{2}}\left(t_{1}\left(x_{1}-t_{1}\right)+\cdots+t_{n}\left(x_{n}-t_{n}\right)\right)^{\alpha_{2}} \\
=2^{\alpha_{2}} \sum_{\substack{\beta \in \mathbb{Z}_{+}^{n} \\
\beta_{1}+\cdots+\beta_{n}=\alpha_{2}}} C_{\alpha_{2}}^{\beta} t^{\beta}(x-t)^{\beta} .
\end{gathered}
$$

Поэтому

$$
\begin{aligned}
I= & (2 \pi)^{2 N} \sum_{\substack{\alpha_{1}+\alpha_{2}+\alpha_{3}=N \\
\beta_{1}+\cdots+\beta_{n}=\alpha_{2}}} C_{N}^{\alpha} C_{\alpha_{2}}^{\beta} 2^{\alpha_{2}} \\
& \times \int e^{2 \pi i x \xi}\left(|t|^{2 \alpha_{1}} t^{\beta} \widetilde{g}(t)\right)\left(|x-t|^{2 \alpha_{3}}(x-t)^{\beta} \widetilde{f}(x-t)\right) d t d x \\
= & \sum_{\alpha_{1}+\alpha_{2}+\alpha_{3}=N} \sum_{\beta_{1}+\cdots+\beta_{n}=\alpha_{2}} C_{N}^{\alpha} C_{\alpha_{2}}^{\beta} 2^{\alpha_{2}} \\
& \times F_{x \rightarrow \xi}^{-1}\left(\int\left(|t|^{2 \alpha_{1}} t^{\beta} \widetilde{g}(t)\right)\left(|x-t|^{2 \alpha_{3}}(x-t)^{\beta} \tilde{f}(x-t)\right) d t\right) \\
= & \sum_{\alpha_{1}+\alpha_{2}+\alpha_{3}=N} \sum_{\beta_{1}+\cdots+\beta_{n}=\alpha_{2}} C_{N}^{\alpha} C_{\alpha_{2}}^{\beta} 2^{\alpha_{2}}\left(D_{\xi}^{\beta} \Delta_{\xi}^{\alpha_{1}} g(\xi)\right)\left(D_{\xi}^{\beta} \Delta_{\xi}^{\alpha_{3}} f(\xi)\right) .
\end{aligned}
$$

Применим эту формулу к $g(\xi)=e^{2 \pi i(x-y) \xi}$ :

$$
\begin{aligned}
& \Delta_{\xi}^{N}\left(e^{2 \pi i(x-y) \xi} f(\xi)\right) \\
& =\sum_{\substack{\alpha_{1}+\alpha_{2}+\alpha_{3}=N \\
\beta_{1}+\cdots+\beta_{n}=\alpha_{2}}} C_{N}^{\alpha} C_{\alpha_{2}}^{\beta} 2^{\alpha_{2}}(2 \pi i)^{2 \alpha_{1}+\beta_{1}+\cdots+\beta_{n}} \\
& \quad \times(x-y)^{\beta}|x-y|^{2 \alpha_{1}} e^{2 \pi i(x-y) \xi} D_{\xi}^{\beta} \Delta_{\xi}^{\alpha_{3}} f(\xi)=e^{2 \pi i(x-y) \xi} \nu(\xi, h) .
\end{aligned}
$$


Относительно $f(\xi)$ получили уравнение в частных производных с постоянными коэффициентами $P(D) f(\xi)=\nu(\xi, h)$. Это эллиптическое уравнение с главным членом $\Delta_{\xi}^{N} f(\xi)$.

Характеристический многочлен дифференциального оператора есть

$$
\begin{aligned}
& e^{-\lambda t} P(D) e^{\lambda t}=P(\lambda) \\
& =\sum_{\alpha_{1}+\alpha_{2}+\alpha_{3}=N} \sum_{\beta_{1}+\cdots+\beta_{n}=\alpha_{2}} C_{N}^{\alpha} C_{\alpha_{2}}^{\beta}(2 \pi i)^{2 \alpha_{1}+\beta_{1}+\cdots+\beta_{n}}(x-y)^{\beta}|x-y|^{2 \alpha_{1}} \lambda^{\beta}|\lambda|^{2 \alpha_{3}} \\
& =|\lambda|^{2 N}+\sum_{\substack{\alpha_{1}+\alpha_{2}+\alpha_{3}=N \\
\alpha_{1} \neq N \neq \alpha_{3}}} \sum_{\beta_{1}+\cdots+\beta_{n}=\alpha_{2}} C_{N}^{\alpha} C_{\alpha_{2}}^{\beta}(2 \pi i)^{2 \alpha_{1}+\beta_{1}+\cdots+\beta_{n}} \\
& \times(x-y)^{\beta}|x-y|^{2 \alpha_{1}} \lambda^{\beta}|\lambda|^{2 \alpha_{3}}+(2 \pi)^{2 N}|x-y|^{2 N} .
\end{aligned}
$$

Соответственно дифференциальное уравнение есть

$$
\begin{aligned}
\left(\Delta_{\xi}^{N}\right. & \left.+(2 \pi|x-y|)^{2 N}\right) f(\xi)+\sum_{\substack{|\alpha|=N \\
\alpha_{1} \neq N \neq \alpha_{3}}} \sum_{|\beta|=\alpha_{2}} C_{N}^{\alpha} C_{\alpha_{2}}^{\beta}(2 \pi i)^{2 \alpha_{1}+\alpha_{2}} \\
& \times(x-y)^{\beta}|x-y|^{2 \alpha_{1}} D_{\xi}^{\beta} \Delta_{\xi}^{\alpha_{3}} f(\xi)=\nu(\xi, h) .
\end{aligned}
$$

Замена переменных $\eta=2 \pi|x-y| \xi, f(\xi)=f(\eta /(2 \pi|x-y|)) \equiv g(\eta)$ дает

$$
\begin{aligned}
\left(\Delta_{\eta}^{N}\right. & +1) g(\eta)+\sum_{\substack{|\alpha|=N \\
\alpha_{1} \neq N \neq \alpha_{3}}} \sum_{|\beta|=\alpha_{2}} C_{N}^{\alpha} C_{\alpha_{2}}^{\beta} i^{2 \alpha_{1}+\alpha_{2}} e_{x-y}^{\beta} D_{\eta}^{\beta} \Delta_{\eta}^{\alpha_{3}} g \\
& =\frac{\nu(\eta /(2 \pi|x-y|), h)}{(2 \pi|x-y|)^{2 N}}
\end{aligned}
$$

где $e_{x-y}=(x-y) /|x-y|$. Заметим, что

$$
\sum_{|\beta|=\alpha_{2}} C_{\alpha_{2}}^{\beta} e_{x-y}^{\beta} D_{\eta}^{\beta}=\left(e_{x-y}, D_{\eta}\right)^{\alpha_{2}} .
$$

Поэтому дифференциальный оператор уравнения принимает вид $Q_{N}(D)=$ $\left(\Delta^{N}+1\right)+q_{2 N-2}(D)$ с младшими членами

$$
q_{2 N-2}(D)=\sum_{\substack{|\alpha|=N \\ \alpha_{1} \neq N \neq \alpha_{3}}} C_{N}^{\alpha} i^{2 \alpha_{1}+\alpha_{2}}\left(e_{x-y}, D\right)^{\alpha_{2}} \Delta^{\alpha_{3}} .
$$

Фундаментальное решение оператора $Q_{N}(D), Q_{N}(D) V_{N}(\eta)=\delta(\eta)$, экспоненциально убывает на бесконечности и имеет в нуле главный член асимптотики, определяемый оператором $\Delta^{N}$, т.е. в окрестности точки $\eta=0$ имеем $V_{N}(\eta)=c_{N} \cdot|\eta|^{2 N-n}(1+o(1))$. Решение уравнения

$$
Q_{N}(D) g(\eta)=\frac{\nu(\eta / 2 \pi|x-y|, h)}{(2 \pi|x-y|)^{2 N}}
$$

можно выписать в виде свертки этого фундаментального решения с правой частью уравнения

$$
g(\eta)=\int V_{N}(\zeta) \frac{\nu((\eta-\zeta) / 2 \pi|x-y|, h)}{(2 \pi|x-y|)^{2 N}} d \zeta .
$$


Соответственно

$$
\begin{aligned}
f(\xi) & =\int V_{N}(\zeta) \frac{\nu((2 \pi|x-y| \xi-\zeta) / 2 \pi|x-y|, h)}{(2 \pi|x-y|)^{2 N}} d \zeta \\
& =\int V_{N}(\tau \cdot 2 \pi|x-y|) \frac{\nu(\xi-\tau, h)}{(2 \pi|x-y|)^{2 N-n}} d \tau .
\end{aligned}
$$

Итак,

$$
\begin{aligned}
C_{\delta}^{\mathrm{opt}}(x, h)= & \int_{\Omega} \varphi(y) \int_{\mathbb{R}^{n}} e^{2 \pi i(x-y) \xi} f(\xi) \Delta_{\xi}^{N}\left(\frac{1}{\left|\mu^{2}(2 \pi i \xi)\right|}[1-\sigma(\xi, \delta)]\right) d \xi d y \\
= & (2 \pi)^{-2 N} \int_{\Omega} \varphi(y) \int_{\mathbb{R}^{n}} e^{2 \pi i(x-y) \xi} \int_{\mathbb{R}^{n}}(2 \pi|x-y|)^{n-2 N} \\
& \quad \times V_{N}(\eta 2 \pi|x-y|) \nu(\xi-\eta, h) \Delta_{\xi}^{N}\left(\frac{1}{\left|\mu^{2}(2 \pi i \xi)\right|}[1-\sigma(\xi, \delta)]\right) d \eta d \xi d y
\end{aligned}
$$

Для гипоэллиптического символа $\mu(2 \pi i \xi)$ из $(2.1)$ имеем

$$
\begin{aligned}
& \left|\Delta_{\xi}^{N}\left(\frac{1}{\left|\mu^{2}(2 \pi i \xi)\right|}[1-\sigma(\xi, \delta)]\right)\right| \\
& \quad \leqslant C_{N} \frac{1}{\left|\mu^{2}(2 \pi i \xi)\right|}\left(\max _{|\xi| \geqslant 1 / h^{\delta}} \frac{1}{(1+|\xi|)^{2 N \rho}}+h^{\delta \cdot 2 N}\right) \leqslant 2 C_{N} h^{2 N \rho \delta} .
\end{aligned}
$$

Полагая $\psi(h)=O\left(h^{\gamma}\right)$ с $\gamma<\rho \delta$, получаем

$$
\begin{aligned}
& \left|C_{\delta}^{\mathrm{opt}}(x, h)\right| \leqslant \mathrm{const} \cdot \frac{h^{2 N \rho \delta}}{\psi(h)^{2 N-n}} \\
& \quad \times\left|\int_{|\xi| \geqslant 2 / h^{\delta}} \int_{\mathbb{R}^{n}} V_{N}(\eta \cdot 2 \pi|x-y|) \nu(\xi-\eta, h) \frac{1}{|\mu(2 \pi i \xi)|^{2}} d \eta d \xi\right| .
\end{aligned}
$$

Внутренний интеграл допускает такую оценку:

$$
\begin{aligned}
\mid \int_{\mathbb{R}^{n}} & \left.V_{N}(\eta \cdot 2 \pi|x-y|) \nu(\xi-\eta, h) d \eta\right|_{\eta=(t+\zeta) / h, t \in \mathbb{Z}^{n}, \zeta \in Q} \\
\quad & =\left|\sum_{t \in \mathbb{Z}^{n}} h^{-n} \int_{Q} V_{N}\left(2 \pi \frac{t+\zeta}{h}|x-y|\right) \nu\left(\xi-\frac{t+\zeta}{h}, h\right) d \zeta\right| \\
& =h^{-n}\left|\int_{Q} \nu\left(\xi-\frac{\zeta}{h}, h\right) \sum_{t \in \mathbb{Z}^{n}} V_{N}\left(2 \pi \frac{t+\zeta}{h}|x-y|\right) d \zeta\right| \leqslant C_{N} h^{-n} .
\end{aligned}
$$

Теперь, чтобы сделать

$$
C_{\delta}^{\mathrm{opt}}(x, h)=o\left(\sqrt{\sum_{s \in \mathbb{Z}^{n} \backslash 0} \frac{1}{\mu(2 \pi i s / h)}}\right),
$$

достаточно взять большое $N$. При соблюдении этого условия мы можем пренебречь членом $C_{\delta}^{\mathrm{opt}}(x, h)$, переходя от оптимальной формулы к асимптотически 
оптимальной. Таким образом,

$$
\begin{gathered}
C^{\mathrm{as}}(x, h)=\int_{\Omega} \varphi(y) \int_{\mathbb{R}^{n}} e^{2 \pi i(x-y) \xi} \frac{\nu(\xi, h)}{|\mu(2 \pi i \xi)|^{2}} \sigma(\xi, \delta) d \xi d y \quad \text { при } \quad \operatorname{dist}(x, \Omega) \geqslant h^{\gamma}, \\
C^{\mathrm{as}}(x, h)=\int_{\Omega} \varphi(y) \int_{\mathbb{R}^{n}} e^{2 \pi i(x-y) \xi} \frac{\nu(\xi, h)}{|\mu(2 \pi i \xi)|^{2}} d \xi d y \quad \text { при } \quad \operatorname{dist}(x, \Omega) \leqslant h^{\gamma} .
\end{gathered}
$$

В варианте $\operatorname{dist}(x, \Omega) \geqslant h^{\gamma}$ возможно уточнение оценки. Благодаря условию $\max _{j}\left|\xi_{j}\right| \leqslant 3 / h^{\delta}$ на носителе $\sigma(\xi, \delta)$ после замены $\xi=t / h+\eta, t \in \mathbb{Z}^{n}, \eta \in Q / h$ остается только слагаемое с $t=0$. Поэтому

$$
\begin{aligned}
C^{\mathrm{as}}(x, h) & =\int_{\Omega} \varphi(y) \int_{Q / h} \frac{e^{2 \pi i(x-y) \eta} \sigma(\eta, \delta)}{\sum_{s \in \mathbb{Z}^{n}}|\mu(2 \pi i \eta) / \mu(2 \pi i(s / h+\eta))|^{2}} d \eta d y \\
& =\int_{\Omega} \varphi(y) \int_{Q / h} e^{2 \pi i(x-y) \eta} \sigma(\eta, \delta)(1-\omega(\eta, h)) d \eta d y
\end{aligned}
$$

c

$$
\omega(\eta, h) \equiv \frac{\sum_{s \in \mathbb{Z}^{n} \backslash 0}|\mu(2 \pi i \eta) / \mu(2 \pi i(s / h+\eta))|^{2}}{1+\sum_{s \in \mathbb{Z}^{n} \backslash 0}|\mu(2 \pi i \eta) / \mu(2 \pi i(s / h+\eta))|^{2}}
$$

Подберем достаточно малое $\delta$ так, чтобы с некоторым $\lambda>0$ было

$$
\begin{aligned}
& \sigma(\eta, \delta)|\mu(2 \pi i \eta)|^{2} \sum_{s \in \mathbb{Z}^{n} \backslash 0} \frac{1}{|\mu(2 \pi i(s / h+\eta))|^{2}} \\
& \quad \leqslant C h^{-2 m^{\prime \prime} \delta} \sum_{s \in \mathbb{Z}^{n} \backslash 0} \frac{1}{|\mu(2 \pi i(s / h+\eta))|^{2}} \leqslant C h^{\lambda} \sqrt{\sum_{s \in \mathbb{Z}^{n} \backslash 0} \frac{1}{|\mu(2 \pi i s / h)|^{2}}}
\end{aligned}
$$

Для этого достаточно взять $\delta<m^{\prime} /\left(2 m^{\prime \prime}\right)$. Тогда

$$
\begin{aligned}
\frac{\nu(\xi, h)}{|\mu(2 \pi i \xi)|^{2}} & =\frac{1}{1+\sum_{s \in \mathbb{Z}^{n} \backslash 0}|\mu(2 \pi i \xi)|^{2} /|\mu(2 \pi i(s / h+\xi))|^{2}} \\
& =1+O\left(\sum_{s \in \mathbb{Z}^{n} \backslash 0} \frac{1}{|\mu(2 \pi i s / h)|^{2}}\right) \cdot|\mu(2 \pi i \xi)|^{2} \\
& =1+o\left(\sqrt{\sum_{s \in \mathbb{Z}^{n} \backslash 0} \frac{1}{|\mu(2 \pi i s / h)|^{2}}}\right)
\end{aligned}
$$

и можно исключить из рассматриваемой формулы $\nu(\xi, h) /|\mu(2 \pi i \xi)|^{2}$. Получаем

$$
C^{\mathrm{as}}(x, h)= \begin{cases}\int_{\Omega} \varphi(y) \int_{\mathbb{R}^{n}} \sigma(\xi, \delta) e^{2 \pi i(x-y) \xi} d \xi d y, & \text { если } \operatorname{dist}(x, \Omega) \geqslant h^{\gamma}, \\ \int_{\Omega} \varphi(y) \int_{\mathbb{R}^{n}} \frac{\nu(\xi, h)}{|\mu(2 \pi i \xi)|^{2}} e^{2 \pi i(x-y) \xi} d \xi d y, & \text { если } \operatorname{dist}(x, \Omega) \leqslant h^{\gamma},\end{cases}
$$


с $\gamma<\rho \delta<\rho m^{\prime} /\left(2 m^{\prime \prime}\right)$. Заметим, что

$$
\begin{aligned}
\int_{\mathbb{R}^{n}} \sigma(\xi, \delta) e^{2 \pi i(x-y) \xi} d \xi \\
\quad=\prod_{j=1}^{n} \int\left(1-\varkappa\left(\left|\xi_{j}\right| h^{\delta}-2\right)\right) e^{2 \pi i\left(x_{j}-y_{j}\right) \xi_{j}} d \xi_{j} \\
\quad=h^{-\delta n} \prod_{j=1}^{n} \int_{|\tau| \leqslant 4}(1-\varkappa(|\tau|-2)) e^{2 \pi i\left(x_{j}-y_{j}\right) \tau / h^{\delta}} d \tau .
\end{aligned}
$$

Функция $\int(1-\varkappa(|\tau|-2)) e^{2 \pi i t \tau} d \tau \equiv T(t)$ принадлежит известному пространству $S$ быстро убывающих функций. Значит,

$$
I=\left|\int_{\mathbb{R}^{n}} \sigma(\xi, \delta) e^{2 \pi i(x-y) \xi} d \xi\right| \leqslant \mathrm{const} \cdot h^{-\delta n} \frac{1}{\prod_{j=1}^{n}\left(1+\left(\left|x_{j}-y_{j}\right|\right) / h^{\delta}\right)^{M}}
$$

с любым $M$. Следовательно,

$$
\begin{aligned}
I & \leqslant \text { const } \cdot h^{-\delta n}\left(\frac{h^{\delta}}{\psi(h)}\right)^{M}=O\left(h^{-\delta n+(\delta-\gamma) M}\right) \\
& =o\left(h^{m^{\prime \prime}}\right)=o\left(\sqrt{\sum_{s \in \mathbb{Z}^{n} \backslash 0} \frac{1}{|\mu(2 \pi i s / h)|^{2}}}\right)
\end{aligned}
$$

при достаточно большом $M$. Поэтому мы положим $C^{\text {as }}(x, h)=0$, если $\operatorname{dist}(x, \Omega) \geqslant h^{\gamma}$ и $\gamma<\delta \rho<m^{\prime} /\left(2 m^{\prime \prime}\right) \cdot \rho$. Таким образом, мы полагаем в формуле $(4.1)$

$$
\psi(h)=h^{\gamma}
$$

Например, для пространства $W_{2}^{m}\left(\mathbb{R}^{n}\right)$ при $|\xi| \leqslant c h^{-\delta}$ справедливо

$$
|\mu(2 \pi i \xi)|^{2} \sqrt{\sum_{s \in \mathbb{Z}^{n} \backslash 0} \frac{1}{|\mu(2 \pi i s / h)|^{2}}} \leqslant \text { const } \cdot h^{-2 m \delta+m} .
$$

Чтобы это было порядка $O\left(h^{\lambda}\right)$ с каким-нибудь $\lambda>0$, достаточно взять $\gamma<$ $\delta<1 / 2$. Значит, можно взять любое $\gamma<1 / 2$.

Итак,

$$
C^{\mathrm{as}}(x, h)= \begin{cases}0 & \text { при } \operatorname{dist}(x, \Omega) \geqslant h^{\gamma} \\ \int_{\Omega} \varphi(y) \int_{\mathbb{R}^{n}} \frac{\nu(\xi, h)}{\left(1+|2 \pi \xi|^{2}\right)^{m}} e^{2 \pi i(x-y) \xi} d \xi d y & \text { при } \operatorname{dist}(x, \Omega) \leqslant h^{\gamma} .\end{cases}
$$




\section{§5. Асимптотически оптимальные коэффициенты кубатурной формулы в случае финитной \\ бесконечно дифференцируемой весовой функции $\varphi$}

Пусть $\varphi \in C_{0}^{\infty}(\Omega)$. За основу опять берем формулу оптимальных коэффициентов

$$
C^{\mathrm{opt}}(x, h)=\int_{Q} e^{2 \pi i \zeta x / h} h^{-n} \frac{\sum_{t \in \mathbb{Z}^{n}} \tilde{\varphi}((t+\zeta) / h) / \mid \mu\left(2 \pi i(t+\zeta) /\left.h\right|^{2}\right.}{\sum_{s \in \mathbb{Z}^{n}} \mid \mu\left(2 \pi i(s+\zeta) /\left.h\right|^{-2}\right.} d \zeta .
$$

Лемма 2. Формула асимптотически оптималъных коэффициентов имеem вид

$$
C^{\mathrm{as}}(x, h)=C^{\mathrm{opt}}(x, h) \cdot(1+o(1))=\varphi(x) \cdot(1+o(1)) .
$$

ДокАЗАтЕльство. Оценим $\widetilde{\varphi}((t+\zeta) / h) ; \widetilde{\varphi}(\xi)$ является элементом пространства $S$ бесконечно дифференцируемых функций, убывающих на бесконечности вместе со своими производными быстрее любой степени $|\xi|$. Поэтому при $t \neq 0$

$$
\left|\widetilde{\varphi}\left(\frac{t-\zeta}{h}\right)\right| \leqslant \text { const } \cdot \frac{1}{(1+|t-\zeta| / h)^{M}}=O\left(h^{M}\right)
$$

с любым $M$. В частности, при $t \neq 0$

$$
\left|\tilde{\varphi}\left(\frac{t+\zeta}{h}\right)\right|=o\left(\sum_{s \in \mathbb{Z}^{n} \backslash 0} \frac{1}{\mid \mu\left(2 \pi i s /\left.h\right|^{2}\right.}\right) .
$$

Значит, для асимптотически оптимальной функции достаточно оставить только слагаемое с $t=0$

$$
C^{\mathrm{as}}(x, h)=\int_{Q} e^{2 \pi i \zeta x / h} h^{-n} \widetilde{\varphi}\left(\frac{\zeta}{h}\right) \frac{1 / \mid \mu\left(2 \pi i \zeta /\left.h\right|^{2}\right.}{\sum_{s \in \mathbb{Z}^{n}} \mid \mu\left(2 \pi i(s+\zeta) /\left.h\right|^{-2}\right.} d \zeta .
$$

Здесь тоже выделим слагаемое с $s=0$ :

$$
\begin{aligned}
C^{\mathrm{as}}(x, h) & =\int_{Q} e^{2 \pi i \zeta x / h} h^{-n} \widetilde{\varphi}\left(\frac{\zeta}{h}\right) d \zeta \\
& -\int_{Q} e^{2 \pi i \zeta x / h} h^{-n} \widetilde{\varphi}\left(\frac{\zeta}{h}\right) \frac{\sum_{s \in \mathbb{Z}^{n} \backslash 0} \mid \mu\left(2 \pi i \zeta /\left.h\right|^{2} / \mid \mu\left(2 \pi i(s+\zeta) /\left.h\right|^{2}\right.\right.}{1+\sum_{s \in \mathbb{Z}^{n} \backslash 0} \mid \mu\left(2 \pi i \zeta /\left.h\right|^{2} / \mid \mu\left(2 \pi i(s+\zeta) /\left.h\right|^{2}\right.\right.} d \zeta .
\end{aligned}
$$

Первое слагаемое есть

$$
\int_{Q} e^{2 \pi i \zeta x / h} h^{-n} \widetilde{\varphi}\left(\frac{\zeta}{h}\right) d \zeta=\int_{Q / h} e^{2 \pi i \eta x} \widetilde{\varphi}(\eta) d \eta=\varphi(x)-\int_{\mathbb{R}^{n} \backslash Q / h} e^{2 \pi i \eta x} \widetilde{\varphi}(\eta) d \eta
$$

Остаток $\int_{\mathbb{R}^{n} \backslash Q / h} e^{2 \pi i \eta x} \widetilde{\varphi}(\eta) d \eta$ равен

$$
o\left(\sqrt{\sum_{s \in \mathbb{Z}^{n} \backslash 0} \frac{1}{|\mu(2 \pi i s / h)|^{2}}}\right),
$$

так как $\widetilde{\varphi} \in S$. Поэтому первое слагаемое в формуле $C^{\mathrm{as}}(x, h)$ заменим на $\varphi(x)$. 
Оценим второе слагаемое. Пусть $\varepsilon \in(0,1)$ удовлетворяет условию

$$
\max _{|\zeta| \leqslant h^{\varepsilon}}\left|\mu\left(\frac{2 \pi i \zeta}{h}\right)\right|^{2}=o\left(\frac{1}{\sqrt{\sum_{s \in \mathbb{Z}^{n} \backslash 0}|\mu(2 \pi i s / h)|^{-2}}}\right),
$$

что выполняется, если

$$
h^{(\varepsilon-1) \cdot 2 m^{\prime \prime}} \sqrt{\sum_{s \in \mathbb{Z}^{n} \backslash 0} \frac{1}{|\mu(2 \pi i s / h)|^{2}}}=o(1),
$$

т.е. надо взять достаточно малое $1-\varepsilon>0$. Тогда дробь, выписанная в формуле второго слагаемого,

$$
\frac{\sum_{s \in \mathbb{Z}^{n} \backslash 0}|\mu(2 \pi i \zeta / h)|^{2} /|\mu(2 \pi i(s+\zeta) / h)|^{2}}{1+\sum_{s \in \mathbb{Z}^{n} \backslash 0}|\mu(2 \pi i \zeta / h)|^{2} /|\mu(2 \pi i(s+\zeta) / h)|^{2}},
$$

не превосходит

$$
o\left(\sum_{s \in \mathbb{Z}^{n} \backslash 0} \frac{1}{|\mu(2 \pi i s / h)|^{2}}\right) .
$$

А для $|\zeta|>h^{\varepsilon}$ используем то, что вся эта дробь меньше 1. Поэтому соответствующая часть интеграла по $\zeta$ допускает оценку

$$
h^{-n} \int_{\substack{\zeta \in Q \\|\zeta|>h^{\varepsilon}}}\left|\widetilde{\varphi}\left(\frac{\zeta}{h}\right)\right| d \zeta \leqslant \text { const } \cdot h^{-n} \int_{\substack{\zeta \in Q \\|\zeta|>h^{\varepsilon}}} \frac{1}{(1+|\zeta / h|)^{M}} d \zeta
$$

с любым $M$.

Очевидно, что при достаточно большом $M$ это есть

$$
O\left(h^{(1-\varepsilon) \cdot M-n}\right)=o\left(\sqrt{\sum_{s \in \mathbb{Z}^{n} \backslash 0} \frac{1}{|\mu(2 \pi i s / h)|^{2}}}\right) .
$$

Таким образом, мы показали, что при $\varphi \in C_{0}^{\infty}$ можно положить

$$
C^{\mathrm{as}}(x, h)=\varphi(x) \text {. }
$$

\section{§ 6. Функция асимптотически оптимальных коэффициентов вне пограничного слоя}

Теперь покажем, что для внутренних узлов, удаленных от границы Г области больше, чем на $\psi(h)$, в асимптотически оптимальной формуле можно полагать значения коэффициентов $c_{k}(h)$ равными $\varphi(h k)$. Действительно, функцию $\varphi \in C^{\infty}(\bar{\Omega})$ через гладкую границу $Г$ можно продолжить на все пространство с сохранением гладкости любых порядков производных вне области. Причем вне некоторого шара $\{x|| x \mid \leqslant R\}$ можно считать $\varphi(x) \equiv 0$.

Для оптимальной кубатурной формулы, выписанной для области $\{x|| x \mid \leqslant$ $R\} \backslash \Omega$, внутренность $\Omega$ будет внешностью. Соответственно, сохраняя асимптотическую оптимальность, можно полагать коэффициенты нулевыми для узлов, 
лежащих внутри $\Omega$ на расстоянии $\psi(h)$ от границы Г. Разность асимптотически оптимальных формул для области $\{x|| x \mid \leqslant R\}$ с $\varphi \in C_{0}^{\infty}(\{x|| x \mid \leqslant R\})$ и области $\{x|| x \mid \leqslant R\} \backslash \Omega$ будет асимптотически оптимальной для области $\Omega$ с $\varphi \in C^{\infty}(\bar{\Omega})$.

Очевидно, ее коэффициенты в узлах из $\Omega$, удаленных от границы $Г$ на расстояние, большее $\psi(h)$, будут равны значениям весовой функции $\varphi(x)$ в этих узлах.

$\mathrm{B}$ частности, для $\varphi(x)=\chi_{\Omega}(x)$ - характеристической функции области $\Omega-$ в этих внутренних узлах коэффициенты равны 1. Таким образом, в этом отношении полученные формулы являются естественными обобщениями кубатурных формул с ограниченным пограничным слоем. Здесь следует отметить, что прежнее определение формул с ОПС (см. [4]) ограничивало толщину пограничного слоя порядком $O(h)$. Пограничный слой наших формул толще, имеет порядок $\psi(h)$. Поэтому изменим определение свойства ОПС, разрешив большую толщину пограничного слоя. Пусть теперь требуется, чтобы толщина пограничного слоя была $o(1)$ при $h \rightarrow 0$. Это сохраняет основные качественные свойства решетчатых формул с ОПС, полезные в приложениях, в частности утверждение об эквивалентности порядковой и асимптотической оптимальностей решетчатых формул с ОПС на семействах $W_{p}^{m}$-пространств (см. [3]).

Предлагаемые формулы являются асимптотически оптимальными на каждом $W_{2}^{\mu}\left(\mathbb{R}^{n}\right)$-пространстве интегрантов, вложенном в $C\left(\mathbb{R}^{n}\right)$.

Сформулируем этот наш основной результат.

Рассматриваются решетчатые кубатурные формулы

$$
\mathscr{K}_{h}(f)=h^{n} \sum_{k \in \mathbb{Z}^{n}} c_{k} f(h k)
$$

приближающие интегралы с весом

$$
\mathscr{I}(f)=\int_{\mathbb{R}^{n}} \varphi(x) f(x) d x
$$

Весовая функция предполагается гладкой в ограниченной области $\Omega$ с гладкой границей $\Gamma: \operatorname{supp} \varphi \subset \bar{\Omega}, \varphi \in \mathbb{C}^{\infty}(\bar{\Omega}), \Gamma \in \mathbb{C}^{\infty}$. Интегранты $f$ принадлежат пространствам $W_{2}^{\mu}\left(\mathbb{R}^{n}\right)$ с нормами

$$
\left\|\left.f\left|W_{2}^{\mu}\left(\mathbb{R}^{n}\right) \|=\sqrt{\int_{\mathbb{R}^{n}}|\widetilde{f}(\xi) \mu(2 \pi i \xi)|^{2} d \xi}, \quad \int_{\mathbb{R}^{n}}\right| \mu(2 \pi i \xi)\right|^{2} d \xi<\infty .\right.
$$

Другие свойства функций $\mu$ перечислены в начале $§ 2$.

Теорема 2. Асимптотически оптимальная решетчатая кубатурная формула с ограниченным пограничным слоем может быть задана такой функцией 
своих коэффициентов $c_{k}^{\text {as }}(h)=C^{\text {as }}(h k, h)$ :

$$
C^{\mathrm{as}}(x, h)= \begin{cases}0 & n p u \operatorname{dist}(x, \Omega) \geqslant h^{\gamma}, \\ \varphi(x) & n p u \operatorname{dist}\left(x, \mathbb{R}^{n} \backslash \Omega\right) \geqslant h^{\gamma}, \\ C^{\mathrm{opt}}(x, h)=\int_{Q} e^{2 \pi i \zeta x / h} h^{-n} & \\ \times \frac{\sum_{t \in \mathbb{Z}^{n}} \tilde{\varphi}((t+\zeta) / h) / \mid \mu\left(2 \pi i(t+\zeta) /\left.h\right|^{2}\right.}{\sum_{s \in \mathbb{Z}^{n}} \mid \mu\left(2 \pi i(s+\zeta) /\left.h\right|^{-2}\right.} d \zeta & n p u \operatorname{dist}(x, \Gamma) \leqslant h^{\gamma},\end{cases}
$$

с любым $\gamma<\rho m^{\prime} /\left(2 m^{\prime \prime}\right)$, где $\rho$ - показатель гипоэллиптичности символа $\mu$ (cм. (2.1)).

Сформулируем этот результат в самом важном частном случае, когда пространство изотропно $\mu(2 \pi i \xi)=\left(1+|2 \pi \xi|^{2}\right)^{m / 2}$, а интеграл невесовой:

$$
\varphi(x) \equiv \chi_{\Omega}(x)= \begin{cases}1, & x \in \bar{\Omega}, \\ 0, & x \neq \Omega .\end{cases}
$$

Теорема 3. Для любых $m>n / 2$ асимптотически оптимальная кубатурная формула с ограниченным пограничным слоем может быть задана функичей коэббициентов: с любым $\gamma<1 / 2$

$$
C^{\mathrm{as}}(x, h)=\left\{\begin{array}{lr}
0 & n p u \operatorname{dist}(x, \Omega) \geqslant h^{\gamma}, \\
1 & n p u \operatorname{dist}\left(x, \mathbb{R}^{n} \backslash \Omega\right) \geqslant h^{\gamma}, \\
C^{\mathrm{opt}}(x, h) & \\
=\int_{\Omega} \int_{Q} h^{-n} \frac{\sum_{t \in \mathbb{Z}^{n}} e^{2 \pi i(x-y)(t+\zeta) / h} /\left(h^{2}+|2 \pi(t+\zeta)|^{2}\right)^{m}}{\sum_{s \in \mathbb{Z}^{n}}\left(h^{2}+|2 \pi(s+\zeta)|^{2}\right)^{-m}} d y d \zeta & n p u \operatorname{dist}(x, \Gamma) \leqslant h^{\gamma} .
\end{array}\right.
$$

\section{§ 7. Упрощение формулы асимптотически оптимальных коэффициентов в пограничном слое}

Упростим формулу асимптотически оптимальных коэффициентов в пограничном слое. Пограничный слой задается условием $\operatorname{dist}(x, \Gamma) \leqslant \psi(h)=o(1)$ при $h \rightarrow 0$. Нам достаточно считать в формуле (6.2) переменные $y$ меняющимися в некоторой малой, но фиксированной (независимо от $h$ ) окрестности $\Gamma_{y}$. Эту окрестность вырежем домножением на соответствующую гладкую ступеньку

$$
\varkappa_{\Gamma}(y)=1-\varkappa\left(\frac{\operatorname{dist}(y, \Gamma)}{\varepsilon}\right)
$$

с "гладкой ступенькой" $\varkappa(t)$ и некоторым $\varepsilon>0$, полагая вместо $\varphi(y)$ произведение $\varphi(y) \cdot \varkappa_{\Gamma}(y) \equiv g(y)$. Функция $g(y)$ совпадает с $\varphi(y)$ в окрестности границы 
$\operatorname{dist}(y, \Gamma) \leqslant C$ и $g(y)=0$ в $\Omega \backslash\{y \mid \operatorname{dist}(y, \Gamma) \leqslant 2 C\}$ (с некоторой постоянной $C)$;

$$
\begin{aligned}
C_{\Gamma}^{\mathrm{opt}}(x, h)= & \int_{\Gamma_{y}} g(y) \int_{Q} h^{-n} e^{2 \pi i(x-y) \zeta / h} \\
& \times \frac{\sum_{t \in \mathbb{Z}^{n}} e^{-2 \pi i y t / h} /|\mu(2 \pi i(t+\zeta) / h)|^{2}}{\sum_{s \in \mathbb{Z}^{n}}|\mu(2 \pi i(s+\zeta) / h)|^{-2}} d \zeta d y \\
= & h^{-n} \int_{Q} \int_{\Gamma_{y}} g(y) e^{2 \pi i(x-y) \zeta / h} \\
& \times \frac{1+\sum_{t \neq 0} e^{-2 \pi i t y / h}|\mu(2 \pi i \zeta / h) / \mu(2 \pi i(t+\zeta) / h)|^{2}}{1+\sum_{s \neq 0}|\mu(2 \pi i \zeta / h) / \mu(2 \pi i(s+\zeta) / h)|^{2}} d y d \zeta .
\end{aligned}
$$

Зададимся вопросом: для каких $\zeta$ сумма

$$
\sum_{s \neq 0}\left|\frac{|\mu(2 \pi i \zeta / h)|}{\mu(2 \pi i(s+\zeta) / h)}\right|^{2}
$$

будет пренебрежимо мала по сравнению с оптимальным порядком функционала погрешности

$$
\nu_{\mathrm{opt}}(h)=\sqrt{\sum_{s \neq 0} \frac{1}{|\mu(2 \pi i s / h)|^{2}}} ?
$$

Дело в том, что для такого множества $\{\zeta\}_{h}$ можно убрать из формулы $C^{\text {opt }}(x, h)$ суммы $\sum_{t \neq 0}$ и $\sum_{s \neq 0}$. Останется

$$
\begin{aligned}
C_{\Gamma, 1}^{\mathrm{as}}(x, h) & =h^{-n} \int_{\{\zeta\}_{h}} \int_{\Gamma_{y}} g(y) e^{2 \pi i(x-y) \zeta / h} d y d \zeta \\
& \equiv \int_{\xi \in\{\zeta\}_{h} / h} \widetilde{g}(\xi) e^{2 \pi i x \xi} d \xi .
\end{aligned}
$$

Например, для $\mu(2 \pi i \xi)=\left(1+|2 \pi \xi|^{2}\right)^{m / 2}$ наше требование означает

$$
\sum_{s \neq 0}\left(\frac{1+|2 \pi \zeta / h|^{2}}{1+|2 \pi(s+\zeta) / h|^{2}}\right)^{m}=o\left(h^{m}\right)
$$

Или $|\zeta / h|^{2 m} h^{2 m}=o\left(h^{m}\right)$. Достаточно, чтобы было

$$
\{\zeta\}_{h}=\left\{\zeta|\exists \varepsilon>0,| \zeta \mid<h^{1 / 2+\varepsilon}\right\} .
$$

В общем случае это будет некоторой окрестностью нуля

$$
\{\zeta\}_{h} \equiv\left\{\zeta|| \zeta \mid \leqslant O\left(h^{\alpha}\right), \text { где }\left|\mu\left(\frac{2 \pi i \zeta}{h}\right)\right|^{2} \sum_{s \neq 0}\left|\mu\left(\frac{2 \pi i(s+\zeta)}{h}\right)\right|^{-2} \leqslant o(1) \cdot \nu_{\mathrm{opt}}(h)\right\} .
$$

Заметим, что наши ранее проделанные вычисления показывают, что можно взять $\alpha=1-\delta$ (см. формулу (4.4)). 
Для $|\zeta| \geqslant C h^{\alpha}$ упрощение формулы коэффициентов в пограничном слое произведем за счет интегрирования по частям по переменной $y$. А именно, воспользуемся формулой Грина

$$
\begin{aligned}
\int_{\Gamma_{y}} g(y) \Delta_{y} f(y) d y & \\
= & \int_{\Gamma_{y}} \Delta_{y} g(y) f(y) d y+\int_{\Gamma} g(y)\left(n_{\mathrm{ext}}(y), D_{y}\right) f(y) d \Gamma \\
& \quad-\int_{\Gamma}\left(n_{\operatorname{ext}}(y), D_{y}\right) g(y) f(y) d \Gamma
\end{aligned}
$$

где $n_{\text {ext }}-$ внешняя единичная нормаль к границе Г области $\Omega$, и будем много раз интегрировать по частям - "перебрасывать" оператор Лапласа $\Delta_{y}$ с множителя

$$
\left.e^{-2 \pi i(t+\zeta) y / h} \equiv \Delta_{y}\left(e^{-2 \pi i(t+\zeta) y / h} \frac{h^{2}}{-|2 \pi(t+\zeta)|^{2}}\right)\right|_{\forall t \in \mathbb{Z}^{n}} \equiv \Delta_{y} f(y)
$$

на $g(y)$. После многократного применения этой формулы интеграл $\int_{\Gamma_{y}}$ можно будет исключить, когда порядок этого слагаемого по $h$ при $h \rightarrow 0$ станет выше $\nu_{\text {opt }}(h)$.

Итак, при $|\zeta| \geqslant C h^{\alpha}$ с $f(y)=e^{-2 \pi i(t+\zeta) / h} h^{2} /\left(-|2 \pi(t+\zeta)|^{2}\right)$ имеем

$$
\begin{aligned}
& C_{\Gamma, 2}^{\mathrm{as}}(x, h)=-\sum_{t \in \mathbb{Z}^{n}} \int_{|\zeta| \geqslant h^{\alpha}} h^{-n} \\
& \times \sum_{j=1}^{J}\left\{\int_{\Gamma}\left[h^{2 j-1}\left\langle n_{\mathrm{ext}}(y), 2 \pi i(t+\zeta)\right\rangle \Delta^{j-1} g(y)-h^{2 j}\left\langle n_{\mathrm{ext}}, D_{y}\right\rangle D^{j-1} g(y)\right]\right. \\
& \left.\quad \times \frac{e^{-2 \pi i(t+\zeta) y / h}}{\left(-|2 \pi(t+\zeta)|^{2}\right)^{j}} \cdot \frac{\left|\mu^{-2}(2 \pi i(t+\zeta) / h)\right|}{\left|\mu^{2}(2 \pi i \zeta / h)\right|^{-1}+\sum_{s \neq 0}\left|\mu^{2}(2 \pi i(s+\zeta) / h)\right|^{-1}} d \Gamma\right\} e^{2 \pi i x \zeta / h} d \zeta .
\end{aligned}
$$

Надо взять $J$ из условия $h^{\delta \cdot 2 J-n}=o\left(\nu^{\text {opt }}(h)\right)$. Например, при $\mu(2 \pi i \xi)=(1+$ $\left.|2 \pi \xi|^{2}\right)^{m / 2}$ получаем $J>(m+n) /(2 \delta)$.

В итоге функция асимптотически оптимальных коэффициентов в пограничном слое $\operatorname{dist}(x, \Gamma) \leqslant h^{\gamma}$ принимает вид

$$
\begin{aligned}
& C^{\mathrm{as}}(x, h)=\int_{|\xi| \leqslant h^{-\delta}} \widetilde{g}(\xi) e^{2 \pi i x \xi} d \xi+h^{-n} \sum_{t \in \mathbb{Z}^{n}} \int_{|\zeta| \geqslant h^{\alpha}} \frac{1}{|\mu(2 \pi i(t+\zeta) / h)|^{2}} \\
& \times \sum_{j=1}^{J} \frac{\int_{\Gamma}\left[e^{-2 \pi i(t+\zeta) y / h} h^{2 j-1}\left\langle n_{\mathrm{ext}}(y), 2 \pi i(t+\zeta)-h D_{y}\right\rangle /\left(-|2 \pi(t+\zeta)|^{2}\right)^{j}\right] d \Gamma}{\sum_{s \in \mathbb{Z}^{n}}|\mu(2 \pi i(s+\zeta) / h)|^{-2}} \\
& \times \Delta^{j-1} g(y) e^{2 \pi i x \zeta / h} d \zeta .
\end{aligned}
$$

Напомним, что здесь $\delta>0$ определяется условием

$$
\left\{\zeta|| \zeta \mid \leqslant h^{1-\delta}\right\} \subset\left\{\zeta|| \mu\left(\left.2 \pi i\left(\frac{\zeta}{h}\right)\right|^{2}=\frac{o(1)}{\sqrt{\sum_{s \in \mathbb{Z}^{n} \backslash 0}|\mu(2 \pi i s / h)|^{-2}}}\right\},\right.
$$


а $J=J(h)-$ условием

$$
h^{\delta \cdot 2 J-n}=o\left(\sqrt{\sum_{s \in \mathbb{Z}^{n} \backslash 0} \frac{1}{|\mu(2 \pi i s / h)|^{2}}}\right) .
$$

Если $\varphi(x)=\chi_{\Omega}(x)$, то в пограничном слое $\left.D^{\beta} g(y)\right|_{\Gamma}=0$ при любой $|\beta| \geqslant 1$ и остается

$$
\begin{aligned}
C^{\mathrm{as}}(x, h) & =\int_{|\xi| \leqslant h^{-\delta}} \widetilde{\varkappa_{\Gamma}}(\xi) e^{2 \pi i x \xi} d \xi \\
- & \frac{i h^{-n+1}}{2 \pi} \sum_{t \in \mathbb{Z}^{n}} \int_{|\zeta| \geqslant h^{1-\delta}} \frac{|\mu(2 \pi i(t+\zeta) / h)|^{-2} \int_{\Gamma}\left\langle n_{\mathrm{ext}}(y),(t+\zeta) /|t+\zeta|^{2}\right\rangle d \Gamma}{\sum_{s \in \mathbb{Z}^{n}}|\mu(2 \pi i(s+\zeta) / h)|^{-2}} \\
& \times e^{-2 \pi i y t / h} e^{2 \pi i(x-y) \zeta / h} d \zeta .
\end{aligned}
$$

Далее рассматриваем только вариант $\varphi(x)=\chi_{\Omega}(x)$.

Мы хотели бы устроить интегрирование по частям, перебрасывая производные на дробь с функциями $\mu$, но этому мешает в первую очередь то, что вместо интеграла у нас стоит сумма по $t \in \mathbb{Z}^{n}$. Поэтому используем конечные разности, сводя дело к преобразованиям Абеля сумм. Введем конечные разности для функции одного переменного

$$
\Delta_{\theta} f(t) \equiv f(t+\theta)-f(t), \quad \bar{\Delta}_{\theta} \equiv f(t)-f(t-\theta)
$$

и для функций $n$ переменных

$$
\begin{gathered}
\Delta_{j, h^{2}} e^{-2 \pi i t y / h} \equiv e^{-2 \pi i\left(t+e^{j} h^{2}\right) y / h}-e^{-2 \pi i t y / h}=e^{-2 \pi i t y / h}\left(e^{-2 \pi i y_{j} / h}-1\right), \\
\bar{\Delta}_{j, h^{2}} e^{-2 \pi i t y / h} \equiv e^{-2 \pi i t y / h}-e^{-2 \pi i\left(t-e^{j} h^{2}\right) y / h}=e^{-2 \pi i t y / h}\left(1-e^{+2 \pi i y_{j} / h}\right), \\
\bar{\Delta}_{j, h^{2} \Delta_{j, h^{2}} e^{-2 \pi i t y / h}} \equiv e^{-2 \pi i t y / h}\left(e^{-2 \pi i y_{j} / h}-1\right)\left(1-e^{+2 \pi i y_{j} / h}\right) \\
\equiv-e^{-2 \pi i t y / h} 4 \sin ^{2}\left(\pi y_{j} h\right) .
\end{gathered}
$$

Значит,

$$
\begin{aligned}
-\sum_{j=1}^{n} \bar{\Delta}_{j, h^{2}} \Delta_{j, h^{2}} e^{-2 \pi i t y / h} & =e^{-2 \pi i t y / h} \sum_{j=1}^{n} 4 \sin ^{2}\left(\pi y_{j} h\right) \\
& =e^{-2 \pi i t y / h} \sum_{j=1}^{n} 4 \pi^{2} h^{2} y_{j}^{2}\left(\frac{\sin \left(\pi y_{j} h\right)}{\pi y_{j} h}\right)^{2} .
\end{aligned}
$$

Отсюда получаем

$$
-e^{-2 \pi i t y / h}=\frac{\sum_{j=1}^{n} \bar{\Delta}_{j, h^{2}} \Delta_{j, h^{2}} e^{-2 \pi i t y / h}}{\sum_{j=1}^{n} 4 \pi^{2} h^{2} y_{j}^{2}\left(\sin \left(\pi y_{j} h\right) /\left(\pi y_{j} h\right)\right)^{2}} .
$$

Наше преобразование Абеля относится к сумме

$$
\sum_{t \in \mathbb{Z}^{n}} \frac{t+\zeta}{|t+\zeta|^{2}} \frac{1}{|\mu(2 \pi i(t+\zeta) / h)|^{2}} \frac{\sum_{j=1}^{n} \bar{\Delta}_{j, h^{2}} \Delta_{j, h^{2}} e^{-2 \pi i t y / h}}{\sum_{j=1}^{n} 4 \pi^{2} h^{2} y_{j}^{2}\left(\sin \left(\pi y_{j} h\right) /\left(\pi y_{j} h\right)\right)^{2}} .
$$


Обозначая

$$
a_{t} \equiv \frac{t+\zeta}{|t+\zeta|^{2}} \frac{1}{|\mu(2 \pi i(t+\zeta) / h)|^{2}}
$$

и временно не учитывая не зависящую от $t$ дробь

$$
\frac{-1}{\sum_{j=1}^{n} 4 \pi^{2} h^{2} y_{j}^{2}\left(\sin \left(\pi y_{j} h\right) /\left(\pi y_{j} h\right)\right)^{2}}
$$

по очереди перекинем конечные разности $\bar{\Delta}_{j, h^{2}} \Delta_{j, h^{2}}$ с экспоненты на $a_{t}$. Получим

$$
\sum_{t \in \mathbb{Z}^{n}} a_{t} \bar{\Delta}_{j, h^{2}} \Delta_{j, h^{2}} e^{-2 \pi i t y / h}=\sum_{t \in \mathbb{Z}^{n}}\left(\bar{\Delta}_{j, h^{2}} \Delta_{j, h^{2}} a_{t}\right) e^{-2 \pi i t y / h} .
$$

Здесь нет граничных членов, так как возникающие в частных суммах $\sum_{\max |t| \leqslant T}$ при переброске отдельных конечных разностей $\bar{\Delta}_{j, h^{2}}$ (или $\Delta_{j, h^{2}}$ ) слагаемые в основном взаимно уничтожаются, а остатки стремятся к нулю при $T \rightarrow \infty$ благодаря абсолютной сходимости рядов $\sum\left|a_{t}\right|, \sum\left(\Delta_{j, h^{2}} a_{t}\right)$.

Применение описанного преобразования Абеля приводит к формуле

$$
\begin{aligned}
& C^{\mathrm{as}}(x, h) \\
& =\int_{|\xi| \leqslant h^{-\delta}} \tilde{\varkappa}_{\Gamma}(\xi) e^{2 \pi i x \xi} d \xi+\frac{h^{1-n}}{2 \pi i} \int_{\Gamma} \int_{|\zeta| \geqslant h^{1-\delta}} \frac{e^{2 \pi i(x-y) \zeta / h}}{\sum_{s \in \mathbb{Z}^{n}}|\mu(2 \pi i(s+\zeta) / h)|^{-2}} \\
& \quad \times \sum_{t \in \mathbb{Z}^{n}} e^{-2 \pi i t y / h} \sum_{j=1}^{n} \frac{\bar{\Delta}_{j, h^{2}} \Delta_{j, h^{2}}\left\langle n_{\mathrm{ext}}(y),(t+\zeta) /|t+\zeta|^{2}\right\rangle}{\sum_{k=1}^{n} 4 \pi^{2} y_{k}^{2}\left(\sin \left(\pi y_{k} h\right) /\left(\pi y_{k} h\right)\right)^{2}} \frac{1}{|\mu(2 \pi i(t+\zeta) / h)|^{2}} d \zeta d \Gamma .
\end{aligned}
$$

Теперь займемся вычислением содержащейся в этой формуле суммы:

$$
\begin{array}{r}
\sum_{j=1}^{n} \bar{\Delta}_{j, h^{2}} \Delta_{j, h^{2}}\left\langle\left(\begin{array}{c}
D y_{n}\left(y^{\prime}\right) \\
-1
\end{array}\right), \frac{(t+\zeta)}{|t+\zeta|^{2}}\right\rangle \frac{|\mu(2 \pi i(t+\zeta) / h)|^{-2}}{\sum_{s \in \mathbb{Z}^{n}}|\mu(2 \pi i(s+\zeta) / h)|^{-2}} \\
\equiv L_{h^{2}}\left[\left\langle\left(\begin{array}{c}
D y_{n}\left(y^{\prime}\right) \\
-1
\end{array}\right), \frac{(t+\zeta)_{n}}{|t+\zeta|^{2}}\right\rangle \frac{|\mu(2 \pi i(t+\zeta) / h)|^{-2}}{\sum_{s \in \mathbb{Z}^{n}}|\mu(2 \pi i(s+\zeta) / h)|^{-2}}\right]
\end{array}
$$

где

$$
\Gamma=\left\{y \mid y_{n}=y_{n}\left(y^{\prime}\right)\right\}, \quad\left(\begin{array}{c}
D y_{n}\left(y^{\prime}\right) \\
-1
\end{array}\right)=n_{\mathrm{ext}} \cdot \sqrt{1+\left(D y_{n}\left(y^{\prime}\right)\right)^{2}} .
$$

Получаем

$$
\begin{aligned}
L_{h^{2}} f(t) \equiv \sum_{j=1}^{n}\left[f\left(t+h^{2} e^{j}\right)-2 f(t)+f\left(t-h^{2} e^{j}\right)\right] \\
=\sum_{j=1}^{n}\left[\int_{0}^{1} \frac{d}{d \sigma} f\left(t+\sigma h^{2} e^{j}\right) d \sigma-\int_{0}^{1} \frac{d}{d \sigma} f\left(t-(1-\sigma) h^{2} e^{j}\right) d \sigma\right] \\
=\sum_{j=1}^{n} \int_{0}^{1}\left[D_{j} f\left(t+\sigma h^{2} e^{j}\right)-D_{j} f\left(t-(1-\sigma) h^{2} e^{j}\right)\right] h^{2} e^{j} d \sigma \\
=\sum_{j=1}^{n} \int_{0}^{1} \int_{0}^{1} \frac{d}{d \tau} D_{j} f\left(\tau\left(t+\sigma h^{2} e^{j}\right)+(1-\tau)\left(t+(\sigma-1) h^{2} e^{j}\right)\right) \cdot h^{2} e^{j} d \tau d \sigma
\end{aligned}
$$




$$
\begin{aligned}
= & \sum_{k=1}^{n} \sum_{j=1}^{n} h^{4} \int_{0}^{1} \int_{0}^{1} e^{k^{*}} D_{k} D_{j} f\left(\tau\left(t+\sigma h^{2} e^{j}\right)+(1-\tau)\left(t+(\sigma-1) h^{2} e^{j}\right) e^{j} d \tau d \sigma\right. \\
= & h^{4} \sum_{k=1}^{n} \sum_{j=1}^{n} e^{k^{*}} D_{k} D_{j} f(t) h^{2} e^{j} \\
& +h^{4} \sum_{k=1}^{n} \sum_{j=1}^{n} \int_{0}^{1} \int_{0}^{1} e^{k^{*}}\left[D_{j} D_{k} f\left(t-(\tau+\sigma) h^{2} e^{j}\right)-D_{j} D_{k} f(t)\right] e^{j} d \tau d \sigma,
\end{aligned}
$$

где $D_{k} D_{j} f$ обозначает оператор взятия второй частной производной, примененный к $f$. Вычисляя его, мы будем каждый раз брать его по $t$ как непрерывной переменной, от $|\mu(2 \pi i(t+\zeta) / h)|^{-2}$ и $(t+\zeta) /|t+\zeta|^{2}$, получая множители $1 / h^{2}$. Остающийся от $h^{4}$ множитель $h^{4} / h^{2}=h^{2}$ "гасится" множителем

$$
\frac{1}{\sum_{k=1}^{n} 4 \pi^{2} h^{2} y_{k}^{2}\left(\sin \left(\pi y_{k} h\right) /\left(\pi y_{k} h\right)\right)^{2}} \approx \frac{1}{4 \pi h^{2}|y|^{2}} .
$$

В итоге получаем

$$
\Delta_{\xi}\left[\left\langle n_{\mathrm{ext}}(y), \frac{\xi}{|\xi|^{2}}\right\rangle \frac{1}{|\mu(2 \pi i \xi)|^{2}}\right] .
$$

А если повторить нашу операцию многократно, то с $|\alpha|=p$ сколь угодно большим получим

$$
\left|D_{\xi}^{\alpha}\left[\left\langle\left(\begin{array}{c}
D y_{n} \\
-1
\end{array}\right), \frac{\xi}{|\xi|^{2}}\right\rangle \frac{1}{|\mu(2 \pi i \xi)|^{2}}\right]\right| \leqslant C_{p} \frac{1}{|\mu(2 \pi i \xi)|^{2}} \frac{1}{(1+|\xi|)^{\rho p}} .
$$

Заметим, что $|\xi| \geqslant 1 / h^{1-\alpha}$, и поэтому итоговый порядок убывания по $h$ можно сделать сколь угодно малым:

$$
O\left(h^{(1-\alpha) \rho p}\right)=o\left(\sum_{s \neq 0} \frac{1}{|\mu(2 \pi i s / h)|^{2}}\right) .
$$

Таким образом, и в общем случае в пограничном слое остается только

$$
C^{\mathrm{as}}(x, h)=\int_{|\xi| \leqslant h^{-\delta}} \widetilde{\varkappa_{\Gamma}}(\xi) e^{2 \pi i x \xi} d \xi .
$$

Заметим еще, что множество $\left\{\xi|| \xi \mid \leqslant h^{-\delta}\right\}$ мы можем заменить на

$$
\left\{\xi\left|\max _{1 \leqslant j \leqslant n}\right| \xi_{j} \mid \leqslant h^{-\delta}\right\},
$$

не нарушая проведенных рассуждений. Итак, получаем функцию асимптотически оптимальных коэффициентов.

Теорема 4. Асимптотически оптимальные коэффиииенты даются формулой

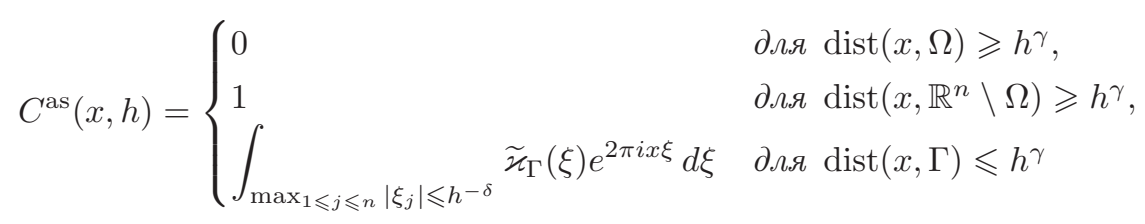


с иГ $(x)$ и любым $\gamma \in(0, \delta)$. Число $\delta \in(0,1)$ выбирается из условия

$$
\int_{\max _{1 \leqslant j \leqslant n}\left|\xi_{j}\right| \leqslant h^{-\delta}}|\mu(2 \pi i \xi)|^{2} d \xi \cdot \sqrt{\sum_{s \in \mathbb{Z}^{n} \backslash 0} \frac{1}{|\mu(2 \pi i s / h)|^{2}}}=o(1) .
$$

Например, для $W_{2}^{m}\left(\mathbb{R}^{n}\right)$-пространств можно взять любые $\delta<1 / 2$ и $\gamma<\delta$.

\section{§ 8. Условия на форму области интегрирования}

Приведенные выше вычисления, завершенные теоремой 4, были сделаны в предположении ограниченности области интегрирования и гладкости ее границы

$$
\Gamma=\left\{x\left|\Phi(x)=0, \Phi \in C^{\infty},\right| D \Phi(x) \mid \neq 0\right\}
$$

В предыдущих вычисления и доказательствах гладкость Г была использована один раз как достаточное условие применимости формулы Грина (7.2). И если вместо гладкости границы Г оставить требование справедливости формулы Грина для самой области интегрирования, то класс доступных $\Omega$ сильно расширяется. Например, в двумерном случае разрешаются все $\Omega$ с кусочно гладкими Г (для больших размерностей нет строгого определения кусочной гладкости границы).

Стремясь к конструктивности вычислительного алгоритма, мы останавливаемся на областях, являющихся пересечением конечного числа ограниченных областей с гладкими границами. Пусть

$$
\begin{gathered}
\bar{\Omega}=\bigcap_{j=1}^{J} \bar{\Omega}_{j} \Subset \mathbb{R}^{n}, \\
\bar{\Omega}_{j}=\left\{x\left|x \in \mathbb{R}^{n}, \Phi_{j}(x) \geqslant 0, \Phi_{j} \in C^{\infty},\right| \Phi_{j}(x)|+| D \Phi_{j}(x) \mid \neq 0\right\}, \\
\Gamma_{j}=\bar{\Omega} \backslash \Omega, \quad \Gamma_{j \varepsilon}=\left\{x \mid \rho\left(x, \Gamma_{j}\right) \leqslant \varepsilon\right\}, \quad \Gamma=\bigcap \Gamma_{j}, \quad \Gamma_{\varepsilon}=\bigcap \Gamma_{j \varepsilon} .
\end{gathered}
$$

Чтобы не углубляться в геометрические тонкости возможных пересечений различных гладких поверхностей, сохраним и требование применимости формулы Грина для $\Omega$. Теперь оно проверяемо для практически встречающихся областей с границами, по-разному, но не очень сложно составленными из гладких кусков. Например, в трехмерном пространстве четырехгранная пирамида является такой областью, и наш алгоритм для нее работает.

Следует отметить, что пересечением конечного числа гладких поверхностей не является, например, поверхность прямого кругового конуса (хотя формула Грина для него сохраняется), т.е. в этом случае наши “общие" ограничения оказываются слишком ограничительными. Их расширение требует дальнейших исследований.

Таким образом, мы приходим к окончательной формулировке основного результата. 
Теорема 5. Кубатурная формула с коэфбициентами (7.5) является асимптотически оптимальной на каждом пространстве $W_{2}^{m}\left(\mathbb{R}^{n}\right)$ с $m>n / 2$ и ненасыщаемой для областей интегрирования (8.1), сохраняющих возможность применения формуль Грина.

В пограничном слое $\operatorname{dist}(x, \Gamma) \leqslant h^{\gamma}$ функиию асимптотически оптимальных коэфбициентов можно задать формулой

$$
C^{\mathrm{as}}(x, h)=\int \varkappa(\Gamma) \prod_{j=1}^{n} \frac{\sin \left(2 \pi\left(x_{j}-y_{j}\right) / h^{\delta}\right)}{\pi\left(x_{j}-y_{j}\right)} d y .
$$

\section{§ 9. Заключение}

Нам удалось получить асимптотически ненасыщаемую формулу. То есть вид коэффициентов не зависит от показателя гладкости - функции $\mu(2 \pi i \xi)$. А в частном случае (наиболее важном) $\mu(2 \pi i \xi)=\left(1+|2 \pi \xi|^{2}\right)^{m / 2}$ и толщина пограничного слоя остается одной и той же для всех $m>n / 2$. И над каждым пространством интегрантов $W_{2}^{m}\left(\mathbb{R}^{n}\right)$ наши формулы коэффициентов дают асимптотически оптимальные решетчатые кубатурные формулы с ограниченным пограничным слоем.

Более того, коэффициенты кубатурной формулы в пограничном слое ведут себя, как в "явлении Гибса". Это означает, что вблизи границы, где $\chi_{\Omega}$ (характеристическая функция области) имеет скачок - разрыв первого рода, она приближается функцией

$$
F^{-1}\left[\prod_{j=1}^{n} \chi_{\left|\xi_{j}\right| \leqslant h^{-\delta}}(\xi)\left(F \chi_{\Omega}\right)(\xi)\right](x),
$$

имеющей равномерно по $h$ ограниченную амплитуду колебаний и не зависящей от параметра $h^{-\delta}$. В частности, эти колебания амплитуд не зависят от гладкостей, заданных пространствами $W_{2}^{m}\left(\mathbb{R}^{n}\right)$, и коэффициенты $c_{k}^{\text {as }}(h) \equiv C^{\text {as }}(h k, h)$ ограничены равномерно по $k$ и $h$.

\section{Список литературы}

[1] С.Л. Соболев, Введение в теорию кубатурных формул, Наука, М., 1974; англ. пер.: S. L. Sobolev, Cubature formulas and modern analysis: An introduction, Gordon and Breach, Montreux, 1992.

[2] С. Л. Соболев, В. Л. Васкевич, Кубатурные формуль, Изд-во ИМ СО РАН, Новосибирск, 1996; англ. пер.: S. L. Sobolev, V.L. Vaskevich, The theory of cubature formulas, Math. Appl., 415, Kluwer Acad. Publ., Dordrecht, 1997.

[3] M. D. Ramazanov, "To the $L_{p}$-theory of Sobolev formulas", Siberian Adv. Math., 9:1 (1999), 99-125.

[4] М. Д. Рамазанов, Теория решетчатых кубатурных формул с ограниченным пограничным слоем, ИМВЦ УНЦ РАН, Уфа, 2009.

[5] К. И. Бабенко, Основы численного анализа, РХД, М.-Ижевск, 2002. 
[6] В.Н. Белых, "О свойствах наилучших приближений $C^{\infty}$-гладких функций на отрезке вещественной оси (к феномену ненасыщаемости численных методов)", Сиб. матем. журн., 46:3 (2005), 483-499; англ. пер.: V. N. Belykh, "On the best approximation properties of $C^{\infty}$-smooth functions on an interval of the real axis (to the phenomenon of unsaturated numerical methods)", Siberian Math. J., 46:3 (2005), $373-385$.

M. Д. Рамазанов (M. D. Ramazanov)

Институт математики с вычислительным центром

Уфимского научного центра РАН

E-mail: ramazanovmd@yandex.ru
Поступила в редакцию 21.05.2012 и 17.09.2012 